A N N A L E S Annales de Bretagne et des Pays de l'Ouest

Anjou. Maine. Poitou-Charente. Touraine

$114-2$ | 2007

Varia

\title{
Un vicomte dans la cité : Jean II de Rohan et Dinan (1488-1516)
}

Laurent Guitton

\section{CpenEdition}

Journals

Édition électronique

URL : http://journals.openedition.org/abpo/63

DOI : $10.4000 / a b p o .63$

ISBN : 978-2-7535-1506-2

ISSN : 2108-6443

Éditeur

Presses universitaires de Rennes

Édition imprimée

Date de publication : 30 juin 2007

Pagination : 7-37

ISBN : 978-2-7535-0510-0

ISSN : 0399-0826

\section{Référence électronique}

Laurent Guitton, « Un vicomte dans la cité : Jean II de Rohan et Dinan (1488-1516) », Annales de

Bretagne et des Pays de l'Ouest [En ligne], 114-2 | 2007, mis en ligne le 31 décembre 2009, consulté le 30 avril 2019. URL : http://journals.openedition.org/abpo/63 ; DOI : 10.4000/abpo.63 


\title{
Un vicomte dans la cité : Jean II de Rohan et Dinan (1488-1516)
}

\author{
Laurent GuITTON \\ Professeur agrégé d'histoire-géographie, \\ Lycée Jean-Rostand - Roubaix
}

En ce matin du 8 août 1488, une rumeur circule dans Dinan assiégée : les autorités de la cité seraient sur le point d'accepter une capitulation. Depuis la défaite catastrophique des troupes bretonnes le 28 juillet dans les landes de Saint-Aubin-du-Cormier, l'inquiétude est générale dans la ville. Dinan serait-elle la prochaine victime des troupes françaises conduites par Louis de la Trémoille, le lieutenant général du roi Charles VIII? L'enceinte urbaine, renforcée dans les années précédentes et tenue par de maigres troupes au service du duc de Bretagne François II, suffirait-elle à contenir la redoutable artillerie française? En cas d'échec de la résistance, la ville ne risquerait-elle pas d'être mise à sac par des centaines de soldats revanchards?

Toutes ces interrogations pesèrent lourdement sur les négociations entre le " très redouté et très puissant seigneur le sire de Rohan ", représentant du roi délégué aux discussions par la Trémoille et " les nobles capitaines et gens d'armes étant à présent à la garde et garnison de ladite ville de Dinan [...] ainsi que les bourgeois, manants et habitants de la dite ville de Dinan représentant la maire et plus saine partie d'iceux ». Les termes du traité permirent probablement de contenter tout le monde : les Dinannais et tous les habitants de l'archidiaconé de Dinan (constitué d'une trentaine de paroisses) obtenaient la garantie de préserver tous leurs biens, de conserver leurs libertés, droits et franchises mais aussi la promesse de ne pas être tirés au sort pour participer au conflit en cours, de se voir restituer hommes et bétail prisonniers des troupes françaises, ainsi que la possibilité de quitter la ville et son pays dans les quinze jours, muni d'un sauf-conduit. En échange, « lesdits capitaines, nobles, bourgeois et habitants de ladite ville bailleront et mettront entre les mains dudit sire de Rohan ladite ville et place de Dinan pour la bailler entre les mains de qui bon lui semblera et y mettre tels gens qu'il lui plaira ${ }^{1}$ ». Le vicomte Jean

1. BARThÉLÉmy, Anatole de, "La capitulation de Dinan en 1488 ", Revue de Bretagne, juillet 1902, p. 20-24; Correspondance de Charles VIII et de ses conseillers avec Louis II de 
II de Rohan faisait ainsi son entrée dans l'histoire de la ville de Dinan, qu'il allait marquer de son empreinte pendant près de trois décennies (1488-1516). Qui est exactement ce personnage ${ }^{2}$ ?

Jean II de Rohan est qualifié de " premier baron de Bretagne" par les États de Bretagne réunis en 1486 : il est donc le deuxième personnage du duché après le duc François II en personne. Non seulement ses domaines couvrent un cinquième du duché (Figure 2), outre quelques possessions dans le royaume de France, mais il est apparenté à la famille des Montfort, détentrice du trône de Bretagne depuis plus d'un siècle. En effet, son grand-père maternel n'est autre que le duc Jean IV (Figure 3); quant à son épouse Marie de Bretagne, elle est elle-même la fille du duc François ${ }^{\text {er }}$ et belle-sœur du duc François II. Jean II de Rohan n'était donc ni plus ni moins que le beau-frère du duc de Bretagne lorsque la guerre fut déclarée en 1487.

Pourtant, les relations entre les

Figure 1 - Portrait de Jean II de Rohan en prière (1452-1516)

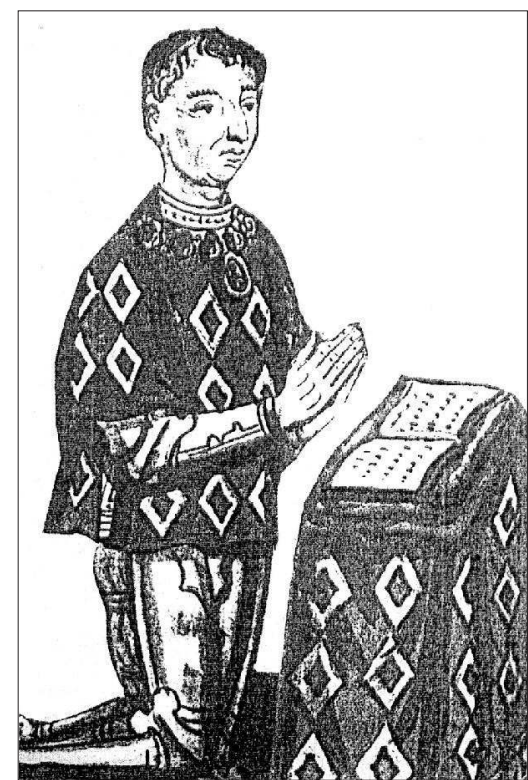
deux hommes furent le plus souvent orageuses. Entre 1470 et 1488, François II confisque à quatre reprises les biens du vicomte et le détient même dans ses geôles, pendant plus de trois ans, pour complicité de meurtre. Il faut savoir que le grand projet de Jean II de Rohan est de faire accéder sa lignée à la couronne ducale et qu'en l'absence d'héritier mâle, il envisage un mariage entre son fils et la fille du duc, la jeune Anne. Il n'hésite donc pas à se rapprocher du roi de France quand il le juge susceptible de l'aider à atteindre son objectif ${ }^{3}$. Telle est

la Trémoille pendant la guerre de Bretagne, 1488, Genève, 1978, p. 208 (" lectres faisans mencions de ce que nostre cousin de Rohan et entre vous avez fait touchant Dignan ").

2. Cette grande figure de l'histoire de la Bretagne à la fin du Moyen Âge a fait l'objet d'une biographie fouillée d'Yvonig GICQUEL, Jean II de Rohan où l'indépendance brisée de la Bretagne, Spezed/Paris, 1994. Néanmoins, l'auteur ne s'est pas appesanti sur le rôle du vicomte à Dinan, ni même sur l'importance de la ville pour les objectifs du vicomte. Les documents $\mathrm{n}^{\circ} 1$ et 2 sont extraits du cahier photographique inclus dans cet ouvrage.

3. NASSIET, Michel, "Fidélités et perspectives dynastiques dans la noblesse bretonne lors de la crise de succession (1470-1491)", dans KERHERVÉ, Jean (dir.), Noblesses de Bretagne du Moyen Âge à nos jours, Rennes, PUR, 1999, p. 103-128. L'auteur démontre la légitimité matrimoniale et politique du projet de Rohan de faire accéder son lignage au trône de Bretagne par un mariage avec la princesse Anne, mais aussi les obstacles qui se sont dressés devant lui, alors qu'il pouvait contribuer à perpétuer l'existence d'un duché de Bretagne indépendant. Le document $n^{\circ} 3$ provient de la page 123 de cet article. 
Article

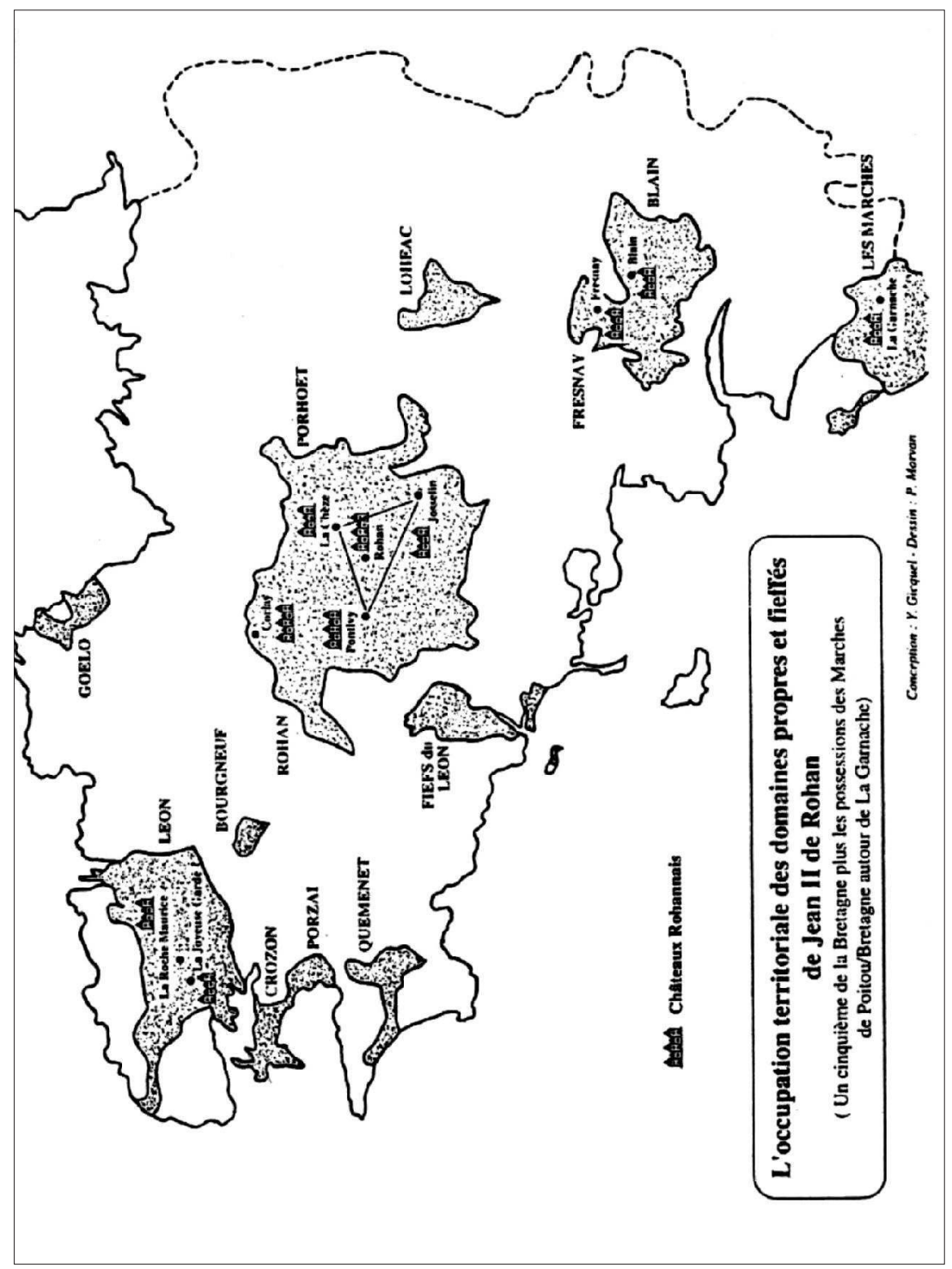

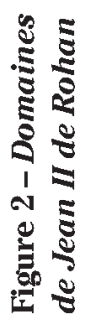


Figure 3 - Alliances entre la maison de Rohan et de Bretagne au $X V^{e}$ siècle

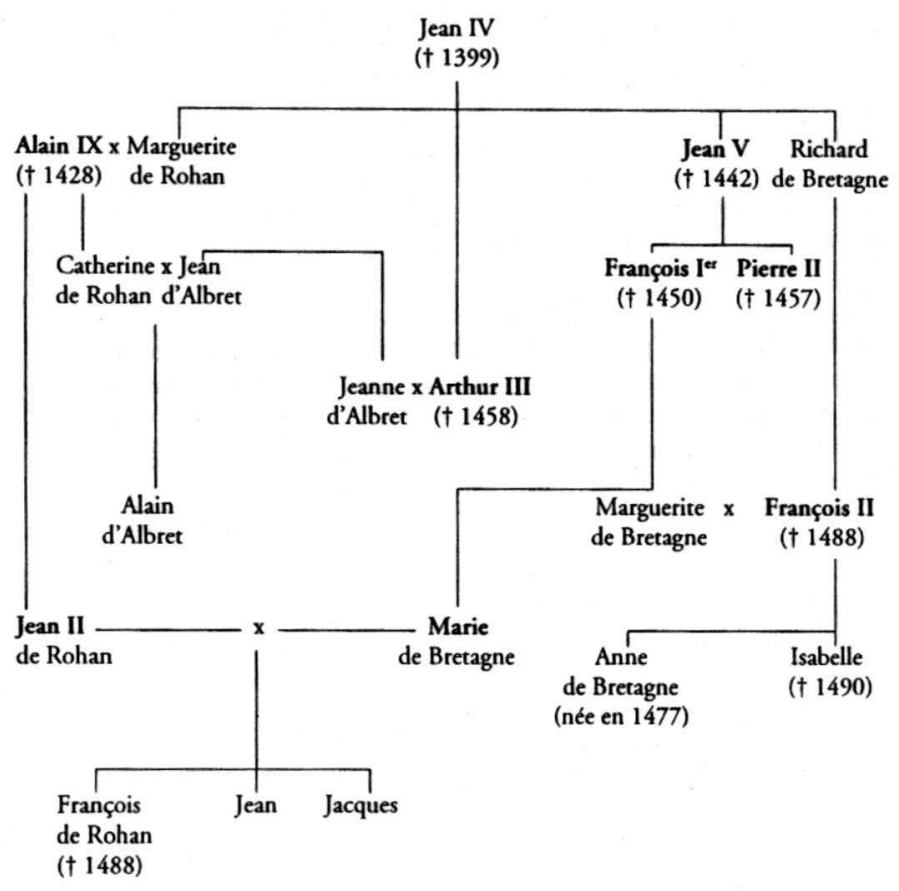

donc la raison de la présence du vicomte au sein des troupes françaises qui encerclent Dinan en ce mois d'août 1488.

L'écheveau des relations tissées entre une communauté et un homme constitue ainsi la trame de notre réflexion. Le protagoniste-clé, c'est le vicomte Jean II de Rohan, l'un des personnages les plus importants de la Bretagne à la fin du Moyen Âge. La communauté, ce sont les habitants de la cité de Dinan, formant une société complexe : clercs et paroissiens, nobles et bourgeois, soldats forains et villageois des alentours, autant d'anonymes qui pour un temps vont renaître à la vie. Avec une population d'environ 5000 habitants, Dinan est alors à son apogée et apparaît comme l'une des principales villes du duché, loin derrière Nantes et Rennes ${ }^{4}$.

Cette relation se développe dans une période décisive, marquée par la guerre d'indépendance et le long processus d'union du duché de Bretagne au royaume de France, à la fin du Xve siècle et au début du XvI ${ }^{\mathrm{e}}$ siècle. En

4. Par sa population, Dinan était de même taille que Vannes et dépassait Saint-Malo sa voisine. Preuve de sa richesse au Xve siècle, elle est régulièrement située au troisième rang des villes qui participent au paiement des aides, impôt versé à l'État breton. LEGUAY, Jean Pierre, " Dinan au temps des ducs de 1283 à 1532 ", dans VILBERT, Loïc-René (dir.), Dinan au Moyen Âge, Dinan, 1986, p. 46-61. 
conséquence, nous croiserons parfois la route de personnages célèbres : le duc de Bretagne François II et sa fille Anne, les rois de France Charles VIII, Louis XII et François I Ir. Mais il s'agit surtout de percevoir les expériences sociales de multiples acteurs, tous liés d'une manière ou d'une autre à Jean II de Rohan, quelles que soient leurs trajectoires et leurs logiques d'intervention : intrigues politiques sur fond de conflit armé, suivies de réconciliations opportunistes; réseau de clientèles privées et groupes de pression municipaux et paroissiaux; détournements de fonds et appels aux dons; concurrence spirituelle et promesse de salut. Autrement dit, un microcosme traversé de multiples tensions politiques, économiques, sociales et culturelles. L'approche locale n'a donc d'intérêt et de sens que dans une logique multi-scalaire, aussi bien régionale que nationale, en mesure d'éclairer les stratégies de tous ces acteurs.

La démarche suivie s'apparente à une véritable enquête historique, parfois proche de la démarche policière, autrement dit " une forme d'élaboration du savoir fondée sur l'interprétation des traces, des indices et des symptômes ${ }^{5}$ ". Elle est de fait largement imposée par le caractère épars de la documentation : trop rares archives encore disponibles, études générales et recherches locales, observation des sites religieux. Puisant à ces sources variées et éclatées, c'est par la confrontation de toutes ces bribes d'expérience qu'il est possible d'accéder "à des logiques sociales et symboliques qui sont celles du groupe, voire celles d'ensemble beaucoup plus larges ${ }^{6}$ ". Pour autant, cette étude aurait difficilement abouti sans une recherche préalable approfondie sur l'histoire de Dinan ${ }^{7}$, ce qui démontre par ailleurs l'intérêt des monographies locales pour la macro-histoire.

Nous nous interrogerons donc sur la relation réciproque entre ce grand personnage de la fin de la période ducale et la ville de Dinan, considérée comme un espace social à la confluence de plusieurs logiques : politique et militaire, économique et financière, religieuse et patrimoniale. En quoi ce mariage - mariage forcé ou de raison? - a-t-il été bénéfique au vicomte et à la cité? Dans quelle mesure Jean II a-t-il réussi à imposer sa mainmise sur les autorités urbaines? Dinan n'était-elle pas qu'un pion de plus dans un jeu de pouvoir complexe, dans une stratégie politique en évolution? Pourquoi avoir tant œuvré à la construction et à l'embellissement du sanctuaire de la paroisse Saint-Malo de Dinan? Finalement, malgré trente ans de domination sans partage sur la ville, pourquoi la figure de Rohan est-elle

5. NoIRIEL, Gérard, Qu'est-ce que l'histoire contemporaine?, Paris, Hachette, 1998, p. 112. Cette " proximité méthodologique entre la démarche heuristique du chercheur médiéval et celle de l'enquêteur " est d'ailleurs relevée par Isabelle DURAND-LEGUERN, " Mener l'enquête au Moyen Âge : le genre du roman policier "médiéval" ", Images du Moyen Âge, p. 197-206, Rennes, PUR, 2006.

6. Revel, Jacques, Jeux d'échelles, Paris, Seuil/Gallimard, 1996, p. 12.

7. L'origine de cette enquête remonte en fait à la fin des années 1980 , lors de la publication de mon mémoire de maîtrise, Étude sur la vie religieuse à Dinan, vers 1400-vers 1580, dir. Hervé MARTIN, 1989, 468 pages (Bibliothèque du CRHISCO, Rennes II ou Bibl. mun. de Dinan). 
tombée dans l'oubli et n'a-t-elle pu trouver sa juste place dans la mémoire contemporaine de la cité ${ }^{8}$ ?

\section{Dinan, théâtre religieux de la rivalité entre Rohan et le duc de Bretagne}

Dans sa stratégie de conquête du trône ducal, tous les moyens sont mis à profit par le vicomte, dès lors qu'ils contribuent à associer son lignage à la famille de Bretagne. C'est d'abord sur le terrain du mécénat religieux qu'il tente de s'arroger les mérites et le prestige du défunt duc, en cherchant à se substituer à lui dans la fondation de sanctuaires religieux. Or, deux opportunités se présentent immédiatement lorsqu'il devient maître de Dinan : la reconstruction de l'église de la paroisse Saint-Malo et la mise en service du couvent des Clarisses.

L'actuelle église Saint-Malo de Dinan est le second sanctuaire de l'histoire de la paroisse. La construction originelle du XI ${ }^{\mathrm{e}}$ siècle était située dans le faubourg Saint-Malo, à l'emplacement de l'actuelle chapelle SaintJoachim. Mais, comme nous l'apprend la lettre signée du duc de Bretagne François II le 10 avril 1488,

" estoit icelle église grande et de bon et somptueux édifice, laquelle en l'an présent par l'avisement et délibération de nos capitaines et gens de guerre, gens de justice, bourgeois et autres notables gens de notre dite ville de Dinan a été démolie et abattue pour obvier aux inconvénients et dommages qui pussent être amenés à notre dite ville et habitants d'icelle et de tout le pays de Dinan si nos ennemis et adversaires les Françoys qui à présent nous font la guerre en notre pays et duché se fussent assiéger devant notre dite ville ainsi qu'ils ont fait devant autres nos villes ${ }^{9}$ ".

On craignait en effet que ce robuste sanctuaire roman ne constituât un bastion idéal pour le bombardement de la ville par les troupes françaises de Charles VIII. Cette destruction était à vrai dire injustifiée au regard des remarquables mutations techniques à l'œuvre dans l'artillerie française ${ }^{10}$; elle s'avéra inutile après la reddition de la ville le 8 août suivant et sans doute source d'inquiétude pour la population locale après le décès de François II le 9 septembre.

Désormais maître de la cité, le "très redouté, haut et puissant Monseigneur Jehan, Vicomte de Rohan et de Léon, Comte de Porhouët et de la Ganasche et seigneur de Beauvoir sur mer " se fait fort de reprendre

8. Ce personnage n'a jamais été mis en valeur par les historiens locaux. MonNiER l'évoque à plusieurs reprises mais préfère détailler l'action du duc de Mercoeur à la fin des guerres de religion. MonNIER, Mathurin, Dinan, mille ans d'histoire, Mayenne, 1977 (2 édition), p. 477-490.

9. Arch. dép. des Côtes-d'Armor, H 432, Prieuré de Saint-Malo, pièce 31.

10. Trois évolutions majeures accrurent l'efficacité de l'artillerie française à la veille du conflit : le canon fondu en bronze, le boulet en fonte et la mobilité des canons montés sur roues. LE PAGE, Dominique et NASSIET, Michel, L'Union de la Bretagne à la France, Morlaix, Skol Vreizh, 2003, p. 81. 
à son compte la promesse du duc de reconstruire l'église paroissiale SaintMalo, cette fois-ci à l'intérieur des remparts et dans le style gothique tardif alors en vogue dans le duché. En effet, il semble que François II n'ait pas eu la possibilité, en cette période de guerre, de verser les sommes nécessaires à l'achat du terrain et au remboursement des propriétaires de biens-fonds. En se substituant au duc, Jean II de Rohan devient de fait le fondateur officiel du sanctuaire, avec tous les droits et devoirs afférents à un tel honneur, empêchant ainsi le défunt duc d'être " participant aux messes, suffrages et oraisons qui y seront célébrés et dits". L'acte de fondation, en date du 12 juin $1489^{11}$, précise que le vicomte débourse plus de cinq cent cinquantesept livres (en monnaie française) pour l'achat des biens-fonds, soit sans doute beaucoup plus que le budget annuel de la paroisse à l'époque. En outre, il offre " la grant vitre du pignon du chanceau d'icelle ", autrement dit le vitrail principal du futur chœur et affirme « son intention de faire aumône et libéralités à ladite paroisse ". En retour, " lesdits paroissiens ont voulu veulent et consentent audit seigneur qu'il soit doteur et fondeur d'icelle église, et qu'il ait enfeux et sépulture s'il le veut au haut du chœur près du grand autel d'icelle église, et autres droits qui appartiennent à fondeur avec qu'il, ses prédécesseurs et successeurs soient participants aux messes, prières et oraisons qui à jamais perpétuellement seront dites et célébrées en ladite église ${ }^{12}$ ".

Les travaux commencent dès l'année suivante, d'abord par le chœur et ses chapelles rayonnantes. En tant que fondateur, Jean II de Rohan avait le loisir d'intervenir dans les choix architectoniques du sanctuaire, au moins dans la partie où il jouissait de ses droits. Or, le type de chevet choisi, constitué de trois chapelles rayonnantes, comprenant trois pignons chacune, rappelle clairement " le parti mis à la mode à la même époque par l'architecte morlaisien Beaumanoir ${ }^{13}$ ". Comment expliquer l'importation d'un modèle de construction religieuse de Basse-Bretagne à Dinan, alors qu'il ne se développe que dans le Trégor, le sud du Léon, le nord-ouest de la Cornouaille ou le pays de Baud dans les décennies suivantes? La comparaison de la localisation des domaines de Rohan (document $n^{\circ} 2$ ) et de la production de ces chevets polygonaux semble aller dans le sens

11. Dom MORICE, Preuves, t. III, collection 641 à 644. Reproduits partiellement par MONNIER, Mathurin, op. cit., p. 115-118.

12. C'est une habitude fréquente du vicomte que de se réserver la maîtresse-vitre des édifices religieux qu'il fonde dans ses domaines, pour y placer armoiries et effigies. PRIGENT, Christiane, Pouvoir ducal, religion et production artistique en Basse-Bretagne, 13501575, Paris, Maisonneuve et Larose, 1992, p. 185-204.

13. Mussat, André, "Les derniers éclats du gothique ", Dinan au Moyen Âge, p. 145. COUfFon, René, "Un atelier architectural novateur à Morlaix à la fin du Xve siècle ", Mémoires de la Société d'Histoire et d'Archéologie de Bretagne, t. XIX, 1938, p. 65-89 a été le premier à signaler ce type de chevet Beaumanoir et à le mettre en rapport avec Jean II de Rohan (p. 86 et 89). Voir plus récemment l'article d'Isabelle BARBEDOR dans CROIX, Alain et VEILLARD, Jean-Yves, Dictionnaire du patrimoine breton, Rennes, Apogée, 2001, p. 125 ainsi que CASTEL, Yves-Pascal, Toute l'histoire de Bretagne, Morlaix, Skol Vreizh, 2003, p. 266 pour le rôle jusqu'à présent occulté de l'architecte Plusquellec dans l'invention de ce style. 
d'une diffusion de ce modèle par Jean II de Rohan, lequel l'aurait proposé, sinon imposé aux trésoriers de la paroisse. Même si Saint-Malo de Dinan ne constitue pas la première tentative de ce nouveau style, elle ne suit que de quelques mois seulement le prototype expérimenté à la chapelle SaintNicolas de Plufur en 1488 par l'atelier Beaumanoir-Plusquellec.

Autrement dit, Rohan est l'initiateur d'une nouvelle esthétique religieuse dans notre ville : il a donc largement contribué à lancer une mode architecturale. Mais ce choix peut aussi bien être envisagé dans une optique politique. Ce nouveau modèle de construction constitue en effet une rupture par rapport à la tradition du chevet plat : or, au cours du Xve siècle, ce dernier style architectural se retrouve dans les nombreux sanctuaires financés par les ducs successifs. À tel point que le chevet plat devient une spécificité de l'art gothique en Bretagne, en grande partie sous l'effet du mécénat ducal ${ }^{14}$. Quel que soit le sens donné à cette innovation de l'abside polygonal, Rohan comptait bien en tirer profit par la multiplication de ces signes héraldiques, afin de bien rappeler sa prééminence dans cet édifice. Outre sa prérogative à placer ses armoiries dans la baie principale de la chapelle axiale du chœur, on peut constater que « les empreintes des armes des Rohan, martelées pendant la Révolution, sont encore visibles sur les côtés antérieurs des piliers du chœur à la retombée des $\operatorname{arcs}^{15}$ ". Mais le vicomte prend soin d'y faire placer les armoiries conjointes de sa famille et de son épouse Marie de Bretagne, "qui sont de 7 macles en champ de gueules, 3, 3, 1, mi-parties de Bretagne ${ }^{16}$ ". Cette association des armes de Rohan et de la dynastie ducale est un moyen pour Jean II de " rappeler l'éminente position généalogique de son épouse ${ }^{17}$ ". Mais ne révèlerait-elle pas aussi la volonté du vicomte de Rohan de rappeler les prétentions de son lignage à la couronne de Bretagne ${ }^{18}$ ?

Pour autant, être mécène d'une église paroissiale, en lieu et place du duc François II, ne peut suffire aux objectifs du vicomte. Il lui faut, si possible, investir le terrain conventuel pour mieux effacer le souvenir du dernier duc dans la mémoire collective des Dinannais. L'affaire de la fondation du couvent des Clarisses de Dinan doit être comprise dans ce contexte. Comme l'a bien montré Hervé Martin dans son étude sur les Ordres mendiants, l'ému-

14. Se reporter à André MuSSAT, "Vitrail et architecture : le fait breton " dans Bretagne. Architecture et identité, Rennes, PUR, 1997, p. 331-337.

15. MonNIER, Mathurin, op. cit., p. 126.

16. Dubuissson-Aubenay, La Bretagne d'après l'itinéraire de Monsieur Dubuisson-Aubenay, Rennes, PUR, 2006, p. 222.

17. NASSIET, Michel, art. cit., 1999, p. 117, note 43.

18. Les armes mi parti Rohan et Bretagne ne se retrouvent par ailleurs que dans le cœur des possessions de Rohan, à savoir le triangle Pontivy - Josselin - Rohan : dans la verrière principale et sur le retable de la Passion de Notre-dame de la Houssaye à Pontivy, dans la baie principale de l'église Saint-Gobrien de Rohan et sur la façade du château de Josselin. Leur présence dans la chapelle Saint-Tugdual de Saint-Guen est peut-être à relier à l'élection de leur fils Claude à l'évêché de Cornouaille en 1501. Prigent, Christiane, op. cit., p. 186; Gatoulllat, Françoise, Hérold, Michel, Les Vitraux de Bretagne, Rennes, PUR, 2005, p. 98-99, 318 et 321 (ill. $n^{\circ} 346$ ). 
lation et la concurrence intra-nobiliaire expliquent la création de nombreux couvents par des familles seigneuriales bretonnes. Ainsi, Alain IX de Rohan est le fondateur du couvent des Franciscains de Pontivy tandis que son fils Jean II est l'instigateur de l'établissement du même ordre à Landerneau, dont les travaux débutent en août 1488. Il est évident que "les Rohan se posent en rivaux directs des ducs sur le terrain du mécénat ${ }^{19}$ ". À Dinan, une autre stratégie se doit d'être développée, dans la mesure où deux couvents de frères prospèrent déjà dans la ville depuis le milieu du XIII ${ }^{\mathrm{e}}$ siècle, grâce à des familles nobles du pays de Dinan : les Jacobins ou Dominicains, fondés par la famille Coëtquen en 1232; les Cordeliers ou Franciscains par les d'Avaugour en 1249.

Or, une opportunité se présente à Rohan. Un projet de création d'un monastère de Clarisses peine à aboutir depuis plusieurs années : une promesse de fondation, et donc de financement, avait bien été faite par le duc François II en personne, mais sa concrétisation se faisait attendre. Maître de la ville, Jean II de Rohan " tente alors de se substituer au défunt duc dans la reconnaissance des Clarisses de Dinan ${ }^{20}$ ". Mais l'affaire est plus compliquée qu'il n'y paraît, le Pape Sixte IV en personne ayant officiellement reconnu le duc comme fondateur du couvent en 1480. En outre, et malgré la lenteur des travaux (suite à des problèmes de délimitation et de remboursement des biens-fonds des particuliers expropriés), la bénédiction, la consécration et la dédicace de l'église du couvent avaient eu lieu en 1484. Ce sont sans doute les difficultés financières du duc et par conséquent la trop lente avancée des travaux qui retardèrent l'installation des sœurs dans l'établissement. Les événements de 1488 allaient en précipiter le dénouement.

Le $1^{\mathrm{er}}$ juillet, le duc fait rédiger un mandement autorisant une dépense de deux cents livres afin d'en terminer avec les conflits de voisinage et d'achever ainsi la construction du monastère. Peu de temps après sont enfin enregistrées par l'évêque de Saint-Malo les bulles et approbations en faveur des dames de Sainte-Claire ${ }^{21}$. Bien qu'il ne soit pas possible de connaître le rôle exact du vicomte Jean II de Rohan dans l'accélération du processus d'installation des sœurs, deux indices nous permettent de penser qu'il avait à cour de se présenter comme un protagoniste de cette fondation. Sa venue à Dinan le 5 décembre, au lendemain de l'inauguration fastueuse du couvent par l'évêque de Saint-Malo en est une première preuve. Une chronologie assez fine s’impose ici pour comprendre le caractère impératif de sa présence. Suite à la capitulation de Dinan du 8 août 1488, Rohan quitte notre ville à une date inconnue, mais nous savons qu'il met le siège devant Guingamp le 20 septembre suivant. « Rohan pensait sans doute que son projet matrimonial serait

19. MARTin, Hervé, Les Ordres mendiants en Bretagne, Rennes, Institut armoricain de recherches historiques, 1975, p. 90-91.

20. PRIGENT, Christiane, op. cit., p. 193.

21. Arch. mun. de Dinan, Clarisses, pièces E 6 bis et E 7. La date exacte de la bulle est malheureusement devenue illisible. 
considéré avec plus d'attention s'il se présentait en position de force ${ }^{22}$. " Le 5 décembre, " le seigneur de Rohan arriva à Dinan, avec l'armée française, qu'il conduisait contre la duchesse Anne sa princesse et proche parente, lequel fit de grands biens au nouveau monastère, à l'aide desquels et de la charité des gens de bien, leur église, cloître, infirmeries, maisons des religieux et sœurs du dehors furent en bref achevées ${ }^{23}$ ". Or, le vicomte repart immédiatement guerroyer en Basse-Bretagne au nom du roi de France. Un tel " détour " par Dinan en pleine guerre suffit à démontrer l'intérêt qu'il porte à cette institution. Un second facteur d'étonnement tient au choix de la responsable du couvent : venue du couvent de Nantes comme ses consoeurs, Catherine Dollo est désignée comme abbesse. Or, elle est présentée comme une femme " de noble race, qui avoit esté élevée près la Dame de Rohan, jusqu'à l'âge de 14 ans, (jusqu'à ce) qu'elle se rendit religieuse à Sainte-Claire de Nantes ${ }^{24}$ ». De telles coïncidences sont troublantes et nous portent à croire qu'à défaut d'avoir pu endosser la reconnaissance et l'honneur de la fondation des Clarisses en lieu et place du duc de Bretagne, Rohan usa de ses richesses et de ses relations personnelles afin d'apparaître comme le principal bienfaiteur de Sainte-Claire.

Quoi qu'il en soit, les sœurs demeurant dans le couvent semblent avoir jugé les générosités de Rohan insuffisantes. Suite à leur requête, elles obtiennent du Pape Innocent VIII une indulgence plénière à perpétuité le 8 mars 1489 " pour favoriser les fidèles qui contribueront à la construction convenable et réparation dudit monastère et donneraient à l'église des livres, calices, ornements, tapisseries [...] aux conditions de visiter, contrits, confessés et communiés l'église du susdit monastère aux fêtes de l'Assomption, de la Nativité, de la Conception et de la Sainte Vierge, et aussi de la fête de Sainte Catherine, vierge et martyre ${ }^{25}$ ". Bien qu'il ne soit pas possible de connaître la chronologie précise de l'itinéraire de Jean de Rohan en cette année $1489^{26}$, des preuves subsistent de ses passages à Dinan autour du 20 avril, soit quelques jours avant la Sainte-Catherine, puis le 12 juin alors qu'il signe le contrat de la fondation de Saint-Malo et peut-être le 2 septembre, soit quelques jours avant la Nativité de la Vierge. Il n'est pas exclu qu'il ait tenu à recevoir ces indulgences pour contribuer à sauver son âme et surtout celle de son fils François, prétendant au trône du duché, tué à la bataille de Saint-Aubin du Cormier un an plus tôt, alors qu'il combattait dans les troupes bretonnes opposées à son propre père.

22. Le PAGE, Dominique et NASSIET, Michel, op. cit., p. 94. Rohan est encore présent à Dinan le 25 août 1488 puisqu'il y rédige un courrier destiné à La Trémoille d'après la Correspondance de Charles VIII et de ses conseillers avec Louis II de La Trémoille durant la guerre de Bretagne, 1488, Genève, 1978, p. 254.

23. Le Grand, Albert, Vies des Saints de la Bretagne-Armorique, Brest/Paris, 1837, p. 180.

24. Ibidem.

25. Arch. mun. de Dinan, Clarisses, pièce E 8.

26. Les quelques indications d'Yvonig GICQUEL, op. cit., nous permettent de retrouver sa trace dans la ville de temps à autre, notamment pendant les années de guerre. 


\section{Jean II de Rohan, bienfaiteur et profiteur de Dinan}

Le vicomte de Rohan apparaît donc comme une bénédiction pour les habitants de Dinan. N'a-t-il pas œuvré à l'installation des sœurs franciscaines dans la ville? N'est-il pas devenu le fondateur de l'église paroissiale Saint-Malo? Certes, ces bonnes actions s'inscrivent dans une stratégie plus globale de puissance et d'affirmation de ses prétentions ducales, alors qu'il convoite toujours le trône de Bretagne. Le roi Charles VIII doit même le ramener à plus de raison. Alors que Rohan mène campagne militaire en Basse-Bretagne, " le Roy envoye ledit seneschal [de Carcassonne] pour dire a mons ${ }^{\mathrm{r}}$ de Roan qu'il ne se die plus duc de Bretaigne, més que s'il y a droit il luy sera gardé. Et le courrier d'ajouter : le Roy prétend droit en la duchié ${ }^{27}$... " À défaut de devenir duc en personne, le vicomte de Rohan espère désormais pouvoir marier son deuxième fils Jean à la princesse Anne, héritière de son défunt père François II.

C'est aussi dans cette perspective qu'il dépense sans compter pour sa bonne ville de Dinan. Si aucun chiffre ne peut être avancé concernant le couvent des Clarisses, certains frais engagés pour l'église paroissiale sont connus. Outre l'achat des terrains de construction en 1489, soit cinq cent cinquante-sept livres tournois, le vicomte débourse deux cents livres en 1493; quatre ans plus tard, il demande à Denis Gervaise, receveur de la ville, de donner aux trésoriers de la paroisse cent livres prélevées sur la recette du domaine " pour commettre et employer à l'édifice et réparation de ladite église $^{28}$ ". Ce qui fait au minimum, d'après les sources avérées, plus de huit cent cinquante livres tournois en dix ans.

Pourtant, si cette somme correspond à une quinzaine d'années de salaires pour un maître maçon, elle n'est guère signifiante pour un seigneur d'une telle envergure. Yvonig Gicquel a comptabilisé que les revenus nets de son domaine personnel rapportaient pas moins de douze mille livres par an et qu'en ajoutant les pensions reçues, soit du duc de Bretagne, soit du roi de France, en fonction des fidélités, il pouvait escompter vingt mille livres de revenus réguliers annuels dans les années 1480 et entre quarante et cinquante mille livres au tout début du $\mathrm{XvI}^{\mathrm{e}}$ siècle! Autant dire que les dépenses somptuaires engagées à Dinan ne représentent que peu de choses au regard de l'immense fortune du vicomte Jean II de Rohan. D'ailleurs, au même moment, comme le montre la figure 4, il fait construire ou agrandir une vingtaine d'édifices religieux, finance l'aménagement de sa forteresse de Pontivy et surtout lance le projet grandiose d'aménagement de la façade du château de Josselin dans un style gothique flamboyant, dont les travaux très coûteux s'étalent de 1490 à $1505^{29}$.

27. Lettre du bailli de la Montagne à La Trémoille en date du 31 octobre 1488, dans Correspondance..., p. 260.

28. MONNER, Mathurin, op. cit., p. 121, note 10.

29. Mussat, André, "Le château de Josselin ", Congrès archéologique de France, Morbihan, 1983, repris dans Bretagne. Architecture et identités, Rennes, PUR, 1997, p. 231-245 et GICQUEL, Yvonig, op. cit., p. 60, 173 et 483 (la carte ${ }^{\circ} 4$ se trouve à la page 426). 
Laurent GuITTON

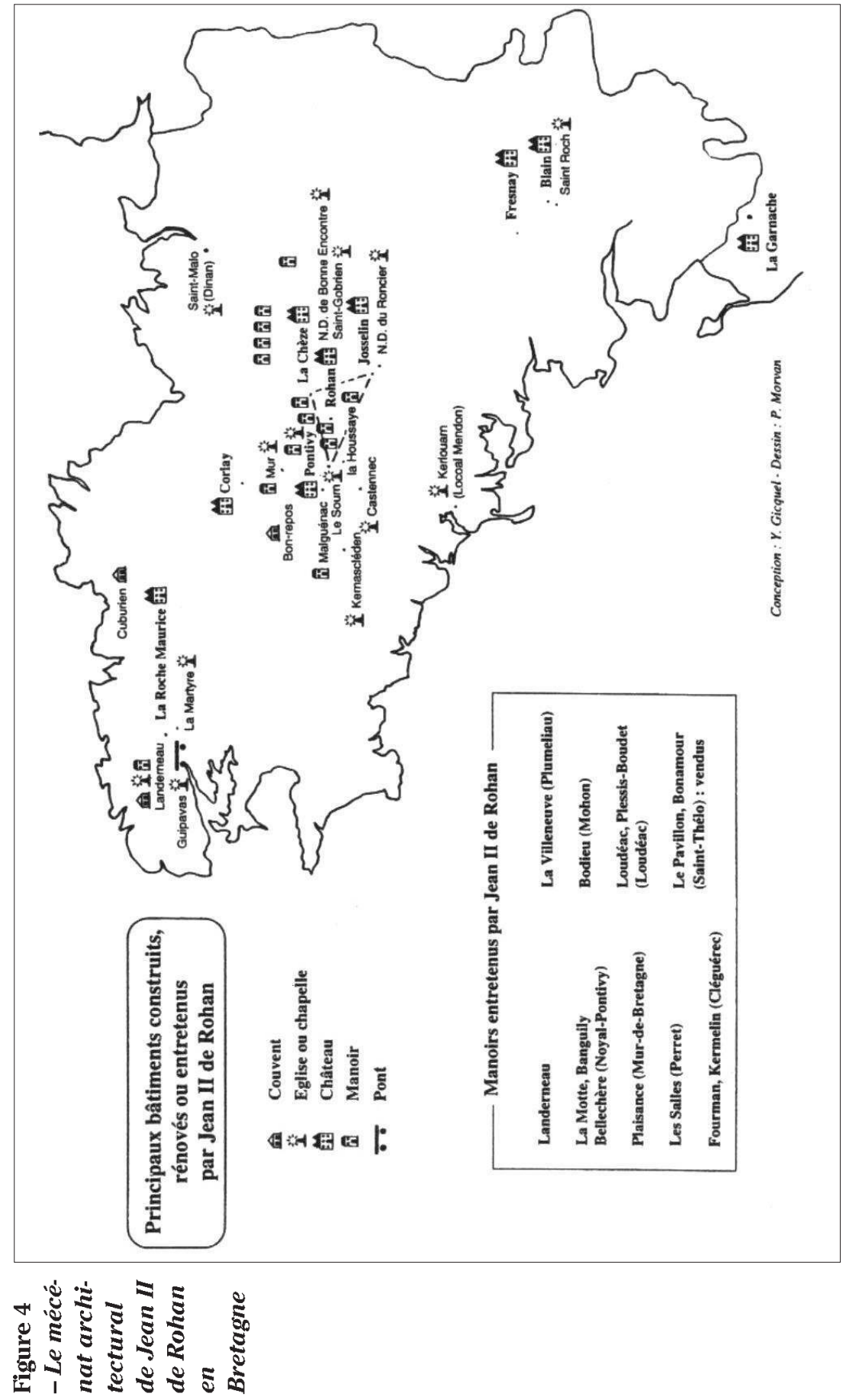


Pourtant, ce n'est même pas sur ses fonds propres que Rohan finance ses largesses dinannaises. Il profite du contexte troublé de la guerre, de son réseau d'hommes de confiance et de la reconnaissance du roi Charles VIII pour son dévouement à la cause française, afin de s'enrichir un peu plus et de financer ainsi son mécénat religieux et politique dans l'ensemble de la Bretagne. Nommé capitaine de la place et de la forteresse de Dinan ainsi que du château voisin de Léhon par le roi en 1488, il détient cette charge militaire suprême et les bénéfices qui en dépendent jusqu'à sa mort en 1516. En outre, la ville de Dinan fait partie de la seigneurie ducale; désormais sous le contrôle des troupes françaises, le roi lui accorde officiellement en 1490 les revenus de la châtellenie de Dinan, lesquels sont gérés par un receveur ordinaire qui doit percevoir les droits à l'intérieur de la ville et dans trente-trois paroisses des alentours ${ }^{30}$. Il est aussi autorisé à prélever à son profit les fouages de l'archidiaconé de Dinan, c'est-à-dire l'impôt direct levé sur tous les foyers roturiers des campagnes de la moitié septentrionale de l'évêché de Saint-Malo. Mais, à vrai dire, Rohan n'a pas attendu une telle autorisation pour s'emparer des revenus liés à la ville et à son pays. En 1490, le roi ne fait qu'officialiser une situation en place depuis 1488, sous prétexte de compenser les dépenses de guerre engagées par Rohan, mais aussi pour le remercier et l'inciter à poursuivre son aide militaire à la France.

Rohan semble d'ailleurs très imbu de ses prérogatives sur la cité. En la seule année 1490, à sa demande, le vicomte ne reçoit pas moins de quatre lettres du roi de France, pour lui confirmer l'ensemble de ses droits ${ }^{31}$. La charte du 8 avril 1491 montre cette préoccupation constante de Jean II de bénéficier de ses privilèges exclusifs, mais aussi de protéger la population dinannaise des exactions des troupes toujours présentes. Charles VIII le confirme :

« Notre cher ami et cousin le Sr de Rohan nous a fait remontré que, après la réduction faite de notre ville et archidiaconé de Dinan en notre obéissance, pour le récompenser de partie des grands frais, mises, pertes et dommages qu'il a eues, soufferts, supportées et endurées pour notre service en la guerre de Bretaigne, nous lui donnâmes et octroyâmes tout le revenu tant ordinaire que extraordinaire de la Seigneurie et archidiaconé dudit Dinan. "

Mais un certain Fédric Darmendaire lui conteste le droit de prélever les " revenus et émoluments des sceaux, greffes et papiers de notre dite ville et archidiaconé de Dinan " et a fait appel à la cour de Paris. Néanmoins, le souverain confirme que Rohan est en droit de jouir des revenus du domaine, " tout ainsi et par la forme et manière que en jouissait le feu duc par avant la réduction de notre dite ville et archidiaconé de Dinan ». Et le roi de poursuivre :

«Et pour ce que notre dit cousin nous a aussi averti que les manants et habitants de notre ville de Dinan se plaignent de ce que nos gens de guerre,

30. Dom Morice, Preuves, t. III, 705 .

31. Médiath. de Nantes, Fonds Bizeul, carton 1696, B 14 : lettres du roi du 17 février, 18 septembre, 28 septembre et 9 octobre 1490 . 
faisant chacun jour le poste en icelle, viennent et exigent sur chacune pipe de vin entrant en ladite ville deux pots de vin, qui sont quatre sols pour pipe et pour chacune pipe qui y en est vingt sols tournois, qui est une grande exaction $^{32}$."

Le roi promet donc à Rohan de remédier à de tels désordres, d'autant plus que par l'acte de capitulation de 1488, Jean II avait promis que " incontinent l'armée du roi qui de présent est devant ladite ville sera retirée ailleurs ". Pourtant, ce n'est qu'à la fin du conflit, en 1492, que Dinan retrouve une vie normale. La place s'avère en effet d'une grande importance stratégique pour le roi : son château et ses remparts permettent de stationner les troupes fraîches et de stocker le ravitaillement qui entrent dans le duché depuis la Normandie ${ }^{33}$. Cette présence militaire offre un autre avantage, celui d'accroître les rentrées financières des trésoriers de Saint-Malo, puisque des quêtes sont régulièrement organisées auprès des soldats, qu'ils soient Français ou Allemands ${ }^{34}$ !

\section{Dinan sous la coupe du réseau Rohan}

La prise de la ville par Rohan en août 1488 se solde par un changement radical de personnel : la plupart des officiers nommés par le duc François II sont révoqués au profit d'hommes de confiance du vicomte ${ }^{35}$ (Figure 5).

Le capitaine en titre, Jean II de Coëtquen, est un haut personnage issu de la grande noblesse : il honore la fonction de Grand Maître de l'Hôtel du Duc depuis 1466 et a reçu le titre de capitaine des villes de Dol, Vannes et Dinan $^{36}$. En son absence, il semble avoir été secondé par Amaury de la Moussaye, seigneur de Dinan. Leur lien remonte peut-être à l'époque où ce dernier était Grand Veneur à l'Hôtel ducal en 1484, donc sous le commandement de Jean de Coëtquen. D'ailleurs, lors de la campagne militaire de l'été 1487, les deux personnages sont dans la forteresse de Dinan avec une armée nombreuse. C'est dans ces circonstances que « le grant maistre de Coëtquen, qui cappitaine estoit de Dinan, demourroit en la ville pour la garde d'icelle, [...] messire Amaury de La Moussaye avec les gens de che-

32. Idem; Jean-Pierre LEguAY, art. cit., Dinan au Moyen Âge, p. 53 et note 85, mentionne déjà dans ce même texte un cas de fraude sur le commerce des vins où sont impliqués des officiers de la garnison de la ville, lesquels vendent du vin sans payer «entrée, bouteillage et autres coutumes et devoirs anciens mis sur le vin qui est descendu, vendu et détaillé en ladite ville".

33. Le roi le reconnaît dans sa lettre du 23 juillet 1488 dans Correspondance..., p. 192.

34. Monnier, Mathurin, op. cit., p. 123.

35. Cette analyse se fonde essentiellement sur les travaux + suivants : KERHERVÉ, Jean, L'État breton aux XIV et XV $V^{e}$ siècles. Les ducs, l'argent et les hommes, Paris, Maloine, 1987 et son catalogue prosopographique dactylographié; LE PAGE, Dominique, Finances et politique en Bretagne au début des temps modernes, 1491-1547, Paris, 1997; LEGUAY, Jean Pierre, art. cit., 1986, p. 46-61 et NASSIET, Michel, « Dictionnaire des feudataires des évêchés de Dol et Saint-Malo en 1480 ", Bulletin de l'Association bretonne, 1990, p. 183-203; 1991, p. 265$296 ; 1992$, p. $221-251$.

36. KERHERVÉ, Jean, op. cit., p. 233-235 (Coëtquen) et p. 257 (La Moussaye). 
val, qui estoient près de trois mil, tireroient à Nantes en diligence pource que ja estoit le bruit que l'armee du roy tiroit celle part ${ }^{37}$ ". Cette armée se fait pourtant surprendre par les troupes françaises près de Châteaubriant; la Moussaye en sort indemne et se réfugie à Nantes. On le retrouve à Dinan l'été suivant... comme premier signataire de la capitulation de la ville, juste après le nouveau capitaine, Jean II de Rohan.

\section{Figure 5 - Le réseau Rohan à Dinan}

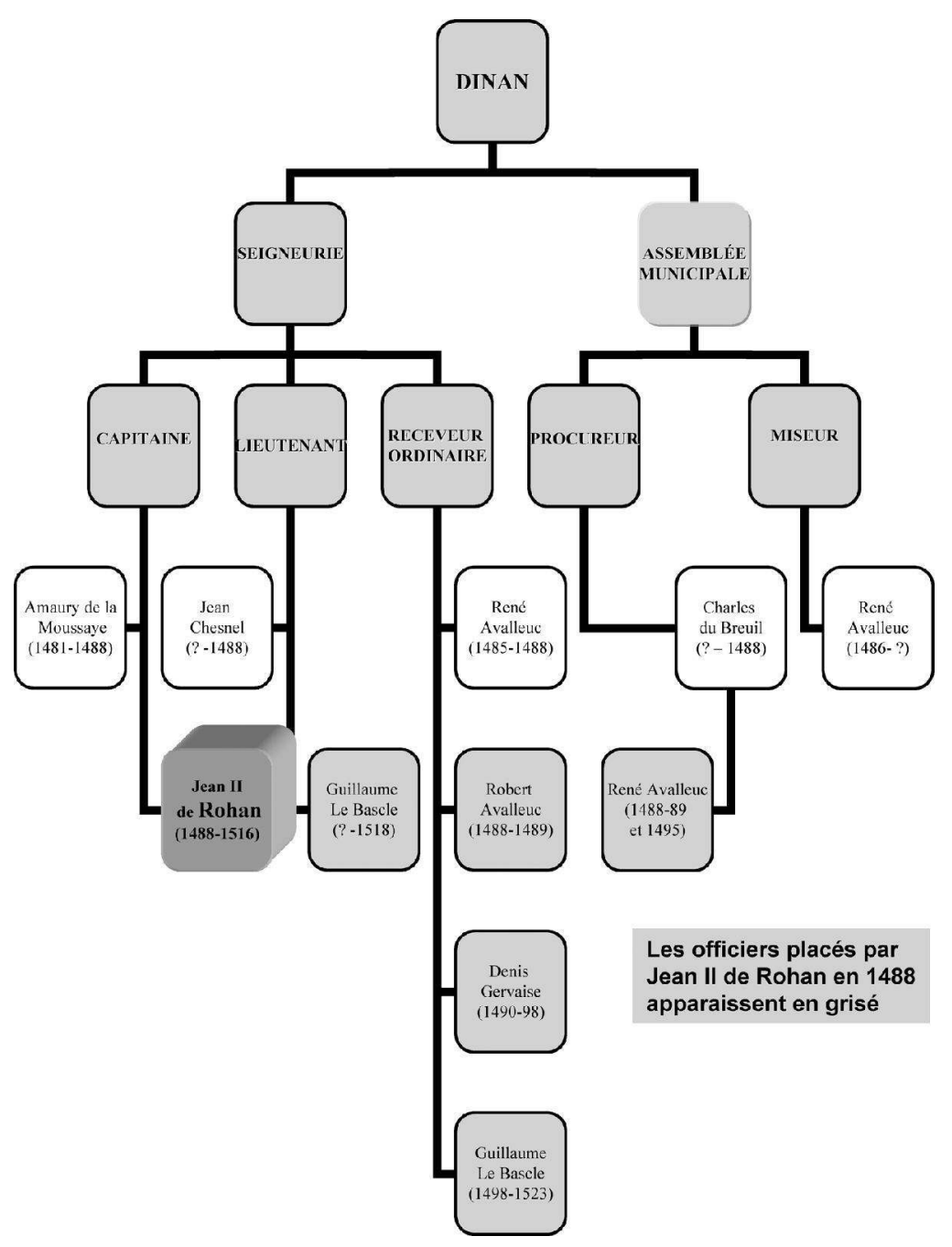

37. BOUCHART, Alain, Grandes chroniques de Bretaigne, Paris, CNRS, 1986, t. II, p. 481. 
En vertu de ce traité et de ses confirmations royales successives, le nouveau capitaine Rohan n'hésite donc pas à placer des hommes déjà sous son patronage et bien intégrés dans les institutions locales : il est nécessaire de s'assurer un contrôle strict de Dinan, de ses édiles comme de ses ressources financières. À cet égard, le cas de René Avalleuc illustre bien les relations de réseau entre les élites locales et les officiers ducaux mais aussi le fonctionnement politico-financier des institutions, reposant sur des pratiques généralisées de corruption et des changements de fidélités.

Issu d'une famille originaire de la région du Josselin-Ploërmel, cœur des fiefs de Rohan, René Avalleuc avait déjà servi le vicomte de Rohan au titre de receveur du comté de Porhoët de 1474 à 1483. En 1485, le duc François II le nomme receveur du domaine de Dinan, grâce à la protection dont il jouissait auprès de certains seigneurs bretons. Ce poste l'incite sans doute à s'installer à demeure dans la paroisse Saint-Malo de Dinan, non sans avoir acquis le manoir du Gué à Saint-Hélen, à une époque où les nobles commencent à disposer d'une résidence d'été à la campagne. Il obtient en 1486 l'office de miseur de la municipalité de Dinan (en charge des comptes municipaux et des grands travaux) et se fait même nommer procureur des bourgeois de la ville, c'est-à-dire maire de la cité, une première fois en 1488, puis de nouveau en 1495. Or, dans l'acte de capitulation d'août 1488, le procureur est encore Charles du Breuil, seigneur de Plumaugat, noble résidant en la paroisse Saint-Malo de Dinan. C'est probablement le nouveau capitaine Rohan qui le fait remplacer par René Avalleuc à la magistrature municipale suprême. La concurrence entre ces deux notables se poursuit d'ailleurs sur le terrain paroissial puisque dans l'acte de fondation de SaintMalo de 1489, sont cités dans l'ordre "Jehan vicomte de Rohan ", suivi de "Charles du Breuil, seigneur de Plumaugat ", puis " René Avalleuc, seigneur de Kerroussaud ", juste avant les deux trésoriers responsables de la gestion de la paroisse. Enfin, l'année précédente, Rohan a confié à Robert Avalleuc (sans doute le frère) le poste de receveur du domaine de Dinan, à la place de René, déjà bien occupé par la charge de procureur de la ville. Mais Robert préfère obtenir un office ducal, peut-être plus gratifiant, mais permettant surtout à la dynastie Avalleuc de maintenir des liens de fidélité envers à la duchesse Anne!

La confiance renouvelée de Rohan à René Avalleuc tient sans doute à une même origine géographique, mais avant tout à un clientélisme familial plus ancien. En effet, Olivier Avalleuc dit " le jeune " était au service du vicomte dès les années 1450, d'abord comme pourvoyeur des vins (14561458), puis en tant qu'argentier (1458-1480), avant d'obtenir la charge de receveur général des domaines du duc de Rohan en 1483-1484. Il est vrai qu'il avait été institué receveur général des terres du vicomte par François II alors que Jean II venait d'être emprisonné par le duc à Nantes. C'est lui qui gérait par conséquent les biens de la famille Rohan, en même temps qu'il levait le fouage de tout l'évêché de Saint-Malo pour le compte du duc François II, non sans assister parfois à certaines délibérations du conseil 
ducal. Un autre membre de ce lignage, " Maître Alain Avalleuc ", apparaît à son tour comme membre de la chambre des comptes des terres et biens de Rohan en tant qu'argentier, puis alloué, c'est-à-dire juge du domaine du vicomte. Il accompagne d'ailleurs le vicomte en cette année 1488, d'abord " vers le roi " de France, et sans doute lors de ses pérégrinations militaires ${ }^{38}$. Les Avalleuc constituent donc une famille digne de confiance aux yeux du vicomte de Rohan, intégrée depuis quelques décennies à son réseau de clientèle, tout en pratiquant parallèlement une politique d'infiltration de l'administration ducale en qualité d'officiers de finances. Rien n'empêche alors un même individu de cumuler offices publics et charges privées, en faveur du duc et au service d'un grand seigneur, quand bien même ceux-ci sont ennemis jurés.

Un tel état de fait incite à se demander dans quelle mesure René Avalleuc, cité parmi les notables de la ville dans l'acte de capitulation de Dinan en 1488, n'a pas été l'un des principaux artisans de la négociation en vue d'une reddition ${ }^{39}$. Ayant été au service du duc de Bretagne comme du vicomte de Rohan, connaissant personnellement Jean II, il était sans doute le mieux placé pour obtenir un compromis acceptable par les deux parties et ainsi épargner un sort funeste à la ville et à ses habitants. Cela expliquerait pourquoi le vicomte mène personnellement les négociations au nom du roi, et non pas Louis de la Trémoille qui se contente d'apposer sa signature sur l'acte de reddition. On peut donc logiquement penser que René Avalleuc a alors reçu en récompense la fonction de procureur de Dinan en cette même année. Il prenait la place de Charles du Breuil, pourtant régulièrement en charge de cette fonction depuis 1463, mais qui avait l'inconvénient d'être le frère aîné de Roland du Breuil, officier ducal resté fidèle à François II.

La constitution de ce réseau d'" agents doubles " permet en outre au vicomte d'utiliser les officiers ducaux pour son unique service, une fois la guerre commencée. À cet égard, Jean Kerhervé a montré que Rohan n'avait pas hésité à mettre en place " une vaste organisation clandestine destinée à financer sur le pays les opérations militaires du parti francophile et à réduire d'autant plus les moyens d'action de l'État breton ". Le montant

38. KeRHERvÉ, Jean, op. cit., Catal., t. II et t. III, p. 454, 644 et 645; GicQuel, Yvonig, op. cit., p. 151, 161, 165, 172, 178-179, 212, 214 et 344; NASSIET, Michel, art. cit., 1990, p. 201.

39. Pour être complet, on doit admettre que plusieurs personnages ont pu avoir une influence sur la réussite de la négociation. Présent à Dinan, Rolland du Breuil, seigneur de Rays en Ploubalay, sénéchal de Rennes et ancien sénéchal de la cité (1480), a pu jouer un rôle non négligeable, puisqu'il a fait partie de l'ambassade envoyée auprès du roi de France par le duc et qui réussit à obtenir une trêve des combats le $1^{\text {er }}$ juin 1488 . (BARTHÉLEMY, Anatole de, art. cit., p. 21; BouchART, Alain, op. cit., t. II, p. 490-491 ; NASSIET, Michel, art. cit., 1991, p. 291). De même, malgré son absence, l'aura de Jean de Coëtquen a pu jouer dans ce dénouement pacifique : proche du duc François II, il est amené à conduire plusieurs ambassades en France, notamment celle de 1488, ce qui lui a valu des pensions du roi de France depuis 1483. En outre, il semble être un ami personnel de Jean II de Rohan qui l'a reçu dans son château de La Chèze en 1486 (Gicquel, Yvonig, $o p$. cit., p. 212, 312; KERHERVÉ, Jean, op. cit., p. 233-235). 
total se serait élevé à de plus de trente mille livres de « détournements de l'impôt public par Jean II de Rohan pendant la guerre d'indépendance ", le tout récolté par « un petit nombre de percepteurs tout à fait régulièrement investis par François II " dans l'ensemble du duché. Parmi eux, il signale un receveur du fouage de l'évêché de Saint-Brieuc en 1488, nommé Denis Gervaise : ce dernier est légitimement récompensé de ses services envers Rohan par l'obtention de la fonction de receveur du domaine de Dinan en 1490, l'année même où la duchesse Anne lance contre lui un ordre d'arrestation, qui ne sera jamais suivi d'effet ${ }^{40}$.

Au final, toutes ces informations incitent à relativiser le coût réel des investissements du vicomte de Rohan à Dinan. Ce n'est donc pas dans sa fortune personnelle qu'il a puisé pour financer son mécénat artistique et religieux. Il a pu en effet utiliser une partie des fonds fiscaux détournés à son profit lors de la guerre de 1487-1491, méthode d'ailleurs cautionnée par le roi. Il a aussi mis à contribution son réseau d'officiers dans la ville, soit par des prélèvements directs sur les revenus du domaine de Dinan, comme on l'observe en 1497, soit en les incitant à contribuer personnellement au financement par des dons. Ainsi, en 1490, un "sieur de Caroussault " (on aura reconnu René Avalleuc, sieur de Keroussaud) fait un don de trentedeux livres, coquette somme pour l'époque correspondant par exemple au salaire d'un maçon pour 160 jours de travail. En 1491, Denis Gervaise (le receveur du domaine de 1490 à 1498) verse un ducat "pour avoir assis la première pierre du pignon ", tandis que $\mathrm{M}^{\mathrm{me}}$ de Plumaugat, femme de Charles du Breuil (le rival malheureux de René Avalleuc) donne douze écus " pour aider à acquérir la perrière de Quelinan (carrière de granit située à Mégrit) et pour avoir deux tombes dedans le cueur de ladite église ${ }^{41}$ ". Mais, au final, ce sont surtout les populations elles-mêmes, celles de Dinan comme de sa région, qui ont financé par leurs diverses contributions fiscales les projets de Jean II de Rohan, à savoir la mise en service du couvent des Clarisses et surtout la construction de l'église paroissiale de Saint-Malo. Juste retour des deniers publics!

\section{Le vicomte et la duchesse : du félon vassal au " très aimé cousin "}

Les relations entre le duc François II et Rohan ont donc été difficiles, ponctuées de fréquents revirements de fidélité de ce dernier, et finalement

40. Kerhervé, Jean, op. cit., p. 591 à 593 et Catal., t. II, p. 480, signale que, comme par hasard, sa charge de receveur du domaine de Dinan prend fin le 8 août 1488, le jour même de la capitulation de la ville! René Avalleuc aurait-il reçu ce même jour la fonction de " maire de Dinan " de l'autorité de Rohan, lequel disposait légalement d'un droit d'intervention dans les affaires municipales? Voir LEGUAY, Jean-Pierre, art. cit., p. 47. Le cas de Denis Gervaise est détaillé par KerhERvé, Jean, op. cit., Catal., t. II, p. 488.

41. MonNIER, Mathurin, op. cit., p. 121-125 a eu accès à des archives aujourd'hui détruites, notamment des comptes de fabrique de la paroisse Saint-Malo. Lors de ma rencontre avec les responsables paroissiaux de Dinan en juillet 1988, en tant que guide de la SPREV dans les deux églises, j'ai appris que des archives de cette paroisse avaient été détruites une vingtaine d'années plus tôt, par négligence semble-t-il. 
d'une opposition finale dans le cadre de la guerre d'indépendance, avec tour jours comme motif central le projet du vicomte de faire accéder son lignage à la Couronne ducale. Suite au mariage de la jeune duchesse Anne avec le roi de France Charles VIII en décembre 1491, l'échec du projet matrimonial de Rohan est patent et, par là même, la possibilité de maintenir une famille bretonne à la tête du duché. C'est sans doute la motivation principale de son implication dans le " complot breton " de 1492, dont l'ambition était de faire appel au roi d'Angleterre afin de chasser les troupes françaises du duché. Suite à ce nouvel échec, Rohan reçoit pourtant le pardon du roi et conserve les fonctions et les biens acquis lors de ces années de conflit. En particulier, il garde le titre de capitaine de Dinan et les revenus réguliers du domaine ducal, dont il continue à nommer lui-même les receveurs; il obtient la reconnaissance de tous les fouages levés pendant la guerre, dont ceux relevant de l'archidiaconé de Dinan, normalement dévolus au souverain ${ }^{42}$.

En revanche, les relations avec la duchesse de Bretagne et reine de France Anne sont marquées du sceau du conflit. Dans sa biographie d'Anne de Bretagne, Georges Minois constate : " Ainsi Jean de Rohan aura-t-il réussi à empoisonner toute l'existence d'Anne de Bretagne, en revendiquant le duché, en cherchant à épouser la duchesse, en intrigant ensuite pour lui donner en mariage son fils aîné, puis son fils cadet, en combattant contre elle du côté du roi, en soutenant le complot de 1492, en lui intentant des procès interminables pour lui arracher le maximum d'avantages ${ }^{43}$. " Cette hostilité réciproque semble néanmoins s'atténuer à partir de 1498, après la mort de Charles VIII et le mariage d'Anne avec Louis XII. Dominique Le Page a d'ailleurs montré que les relations entre Anne et l'ensemble de la noblesse bretonne connaissent une phase d'apaisement entre 1498 et 1514, poursui-

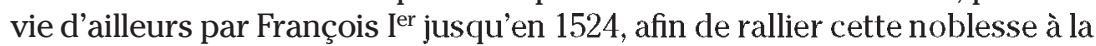
cause royale ${ }^{44}$. Alors que la duchesse Anne vient de reprendre le contrôle de l'administration bretonne, elle confirme à Rohan ses droits sur la ville le 16 octobre 1498 : « De la part de notre très cher et très aimé cousin le seigneur de Rohan nous a été exposé que il a eu et obtenu de nous lettres de don des capitaineries de Dinan et du chastel de Léhon, ensemble du revenu de la seigneurie et archidiaconé de Dinan ». De même, Anne valide le choix de Guillaume Le Bascle comme receveur ordinaire de Dinan " à la requeste de monseigneur de Rohan ${ }^{45}$ ".

42. Médiath. de Nantes, Fonds Bizeul, carton 1696, B 14, actes du 14 septembre et 26 novembre 1494. Arch. Dép. de Loire-Atlantique, B 51 f ${ }^{\circ} 13$, confirmé le 22 octobre 1494 $\left(f^{\circ} 16\right)$. En outre, Rohan obtient de Charles VIII vingt mille livres de pension annuelle, encore en cours en 1504. Le PAGE, Dominique, op. cit., p. 22, 87 et 181.

43. MinoIs, Georges, Anne de Bretagne, Paris, Fayard, 1999, p. 366.

44. Le PAGe, Dominique, " Noblesse et pouvoir royal en Bretagne (1480-1540)" dans KERHERVÉ, Jean (dir.), Noblesses de Bretagne du Moyen Âge à nos jours, Rennes, PUR, 1999, p. 129-149.

45. Arch. dép. de Loire-Atlantique, B 51 f 84 et E 128/22; LE PAGE, Dominique, op. cit., p. 10 et 665 . 
Il est vrai que quelques jours avant, en septembre 1498, la jeune veuve Anne fait un premier passage dans la cité dinannaise. D'après le chroniqueur Alain Bouchart, " en ce temps elle alla à Dynan où elle fut plus assidue aux oraisons et ne voulut ni festes ni ébats ${ }^{46}$ ". Se contenta-t-elle de prier pour l'âme de son défunt époux ou fit-elle aussi des dons aux institutions religieuses de la ville? Rien ne permet de le dire. La visite de 1505 est un peu mieux connue. C'est un véritable tour de Bretagne qu'effectue la souveraine dans un duché qu'elle ne connaît finalement que très peu, escortée par le vicomte de Rohan ${ }^{47}$. Ils sont reçus à Dinan le 19 septembre : le capitaine revient donc dans sa place forte, accompagnée de la reine-duchesse, qui l'a confirmé dans ce titre quelques années plus tôt. Malheureusement, on ne connaît que les conditions de la réception du « couple " princier aux abords de la ville, grâce au témoignage du sacristain du baron de la Hunaudaye, Olivier de la Roche.

" De la Hunaudaye, madame Anne alla voir sa bonne ville de Dinan forte et puissante ville et de grande défense; auquel lieu vint au devant d'elle environ demie lieue une bergerie fort joyeuse à la collaudation de ladite dame, faite de haut style et jouée à l'advenant par gens de sorte et fut reçue ladite dame comme maîtresse et principale de son duché et lui fut faite joyeuse chaire de ses bons vins d'Anjou ${ }^{48}$."

Cette entrée princière, organisée par les bourgeois de la ville, s'adressait sans doute autant au vicomte qu'à sa suzeraine. Bien qu'une lettre du roi Louis XII la presse de revenir à ses côtés ${ }^{49}$, elle prend soin de faire un don de cent livres à la paroisse Saint-Malo. Enfin, malgré un très bref séjour dans le duché en juin 1507, Anne effectue pourtant un dernier passage à Dinan : elle est désignée marraine de la nouvelle cloche du beffroi tandis que le vicomte, également présent, en est le parrain ${ }^{50}$. L'année suivante, elle s'engage à verser la somme de cent livres pendant dix ans, tandis que le roi donnera la même somme en 1511. Au total, le couple royal a contribué à hauteur de mille deux cents livres à l'édification de l'église, soit peut-être plus que Rohan, le fondateur officiel.

Dans ce contexte, comment appréhender cet apport d'Anne et de son mari au financement de l'église paroissiale de Saint-Malo? Faut-il y voir une volonté de concurrencer Jean de Rohan dans son acte de fondation? Anne, réputée pour son obstination tenace, devait savoir que la promesse de reconstruction du sanctuaire paroissial fut d'abord faite par son père

46. MONNIER, Mathurin, op. cit., p. 276-277.

47. MÉTAIREAu, Rachel, Anne de Bretagne visite son duché en 1505, Mémoire de maîtrise, dactyl., Univ. de Nantes, 1999, p. 29, 32, 48, 50, 60 et 64. L'évêque de Saint-Malo rejoint la reine au cours du périple, peut-être à Dinan, ultime étape avant sa cité épiscopale. Dom Morice, Preuves, t. III, coll. 869.

48. Bellevue, La Baronnie de la Hunaudaye, Rennes, 1908 et MonNIER, Mathurin, op. cit., p. 277.

49. MiNOIS, Georges, op. cit., p. 488-495.

50. MalherBe, Gérard, « Anne pour vray, je fus nommée... », Le Pays de Dinan, t. III, 1983, p. $53-62$. 
François II. Ce serait alors la réponse de la reine-duchesse au vicomte, après sa tentative de se substituer à son père comme fondateur du couvent des Clarisses. Ou bien s'agit-il tout simplement de contribuer à l'embellissement de la cité, sans arrière-pensée politique et sans volonté de conflit envers le vicomte?

Quoi qu'il en soit, les débuts de l'histoire de l'église paroissiale SaintMalo de Dinan s'intègrent dans un jeu politique subtil et significatif. Construction promise par le duc François II en 1488, reprise à son compte et inaugurée par le vicomte de Rohan en 1489, financée partiellement par ce dernier grâce aux recettes du domaine ducal de la ville et au fouage du pays de Dinan, elle a amplement bénéficié des largesses de la duchesse Anne et du roi de France. Difficile de trouver mécènes plus prestigieux pour une simple église paroissiale!

\section{Jean II de Rohan renié par les Dinannais?}

Simple église? Ce n'est pas l'avis des paroissiens en ce mois de mai 1518, alors qu'ils en appellent à nouveau à la générosité du roi de France François I ${ }^{\mathrm{er}}$. La duchesse Anne s'est éteinte en 1514, suivie par Jean de Rohan en 1516 : nos paroissiens sont donc à la recherche d'un nouveau donateur. Mais laissons plutôt la parole aux notables réunis à la cour de Dinan, à savoir six bienfaiteurs nobles " circonvoisins de ladite ville de Dinan ", le procureur et le lieutenant, autorités politique et militaire suprêmes, sans doute le curé de la paroisse et six bourgeois fortunés, tous marchands.

"Ont été présentes lesdites personnes et interrogées à la supplication et requête des trésoriers touchant l'état de l'église neuve encommencée à faire édifier en ladite ville par les paroissiens d'icelle et autres leurs amis et bienfaiteurs ainsi que ensuit [...]. Lesquels m'ont dit et recordé et certifié et relaté avoir et qu'ils ont eu connaissance de ladite ville de Dinan dès le temps de leur enfance que, au faubourg d'icelle à distance de la clôture de la ville d'environ deux [?] de boulet, y avait d'antiquité une grande belle et somptueuse église paroissiale nommée et vulgairement appelée l'église paroissiale de Saint Malo de Dinan; et de la grande magnificence dont elle était et crainte que ladite ville n'en eut été endommagée par les ennemis de ce pays, fut abattue et rasée par terre durant le temps de la guerre qui fut dans le temps d'environ trente ans. Et que depuis ont commencé à construire et édifié dès le temps de ladite guerre une autre église paroissiale en ladite ville, quel édifice d'église est encommencé et entrepris, a été fait par les paroissiens d'icelle paroisse de Saint Malo en telle sorte et manière et si somptueusement que l'on vit, et est une chose toute notoire que ce sera l'une des plus belles églises paroissiales de ce dit pays pourvu qu'elle soit conduite et maintenue ainsi que l'on y a commencé; disant lesdits nommés impossible chose être audits paroissiens qui sont pauvres de la pouvoir achever si ce n'était avec l'aide du roi et du notre souverain et lequel et ses prédécesseurs, que Dieu absolve, y ont donné et aumôné de leurs deniers qui ont été bien et loyalement employés ainsi bien que apparaît par ledit somptueux édifice; et si lesdits pauvres paroissiens n'ont aide de notre dit souverain, ladite église 
demeurerait imparfaite et en ce que en a de fait pourrait tomber en ruine et décadence qui serait la totale perdition dudit édifice ${ }^{51}$."

Ce texte appelle plusieurs commentaires. D'abord, ces notables se présentent comme les garants de la mémoire paroissiale mais nous ne devons pas nous laisser abuser par leur émouvante évocation historique. L'exagération de rigueur vise à mettre en valeur la grandeur et la splendeur de l'ancien sanctuaire, pour mieux dramatiser la lenteur des travaux du nouvel édifice, car il est clair que l'église, en particulier le chœur, était déjà bien avancée. Ils le reconnaissent d'ailleurs implicitement lorsqu'ils vantent le fait qu'elle sera la plus belle église de la région, affirmant ainsi leur chauvinisme paroissial. Ils visaient directement les voisins de la paroisse Saint-Sauveur, également engagés dans de lourds travaux d'agrandissement depuis les années 1480, mais qui n'avaient pas la chance de bénéficier d'un mécénat aussi prestigieux.

Ensuite, on peut s'étonner que le fondateur Jean de Rohan ne soit pas une seule fois mentionné, d'autant plus que, dans la liste des notables cités, après " Maître Guy de Saint-Cyre, procureur des bourgeois ", on retrouve le lieutenant Guillaume Le Bascle! Or, ce Le Bascle est un homme d'influence dans la cité, ayant appartenu au réseau Rohan : on se souvient qu'il est devenu le receveur ordinaire du domaine de Dinan à la demande expresse de Jean II de Rohan en 1498 et l'est resté pendant 25 ans ${ }^{52}$. Sieur des Alleux, ayant accédé à la noblesse, relevant de la paroisse voisine de Saint-Sauveur, il est aussi membre laïc, avec son épouse, de la très convoitée "Confrérie des prêtres et chapelains de Dinan ${ }^{53}$ ". En ce jour, c'est donc au titre de lieutenant de la cité qu'il est présent, autrement dit second personnage militaire derrière le capitaine : peut-être fut-il même nommé à ce poste par le vicomte lui-même, afin de pallier à son absence chronique de la ville? Deux ans seulement après sa mort, le vicomte de Rohan semble donc déjà ne plus compter pour les paroissiens, malgré quasiment trente années à la tête de la cité et la présence de son fidèle client. Le silence de Le Bascle estil signe d'ingratitude ou a-t-il dû se plier à la volonté des hommes influents de la paroisse? L'absence de toute mention au fondateur Rohan peut être interprétée comme une volonté délibérée d'occulter une référence désormais inutile et ainsi de mieux valoriser le mécénat royal. Cela pourrait aussi expliquer la volonté d'insister sur les donations de ses prédécesseurs au profit de la paroisse, en particulier à l'époque de la duchesse Anne et de ses époux.

51. Arch. dép. des Côtes-d'Armor, H 432 (Prieuré de Saint-Malo), pièce 33. Pour une meilleure compréhension, le texte a été partiellement corrigé et modernisé.

52. En 1515, François $1^{\mathrm{er}}$ avait en effet reconduit Rohan dans ses fonctions dinannaises et, par la même, Guillaume Le Bascle dans celle de receveur, gardant la confiance du vicomte depuis déjà dix-sept ans... Arch. dép. de Loire-Atlantique, B 51 f ${ }^{\circ} 172$. Voir LE PAGe, Dominique, op. cit., p. 526 et 527.

53. Bibl. mun. de Dinan, Série A (Saint-Sauveur, pièce datée de 1515) et série B (Confrérie des prêtres et chapelains, trois registres annuels de 1509, 1511 et 1517). 
Enfin, cette requête ne se fait pas n'importe quand. En cette année 1518, François I $^{\text {er }}$ fait le tour de son royaume et les notables dinannais ont dû avoir vent de son passage prévu en Bretagne à l'été ${ }^{54}$. Afin de prendre les devants, les autorités municipales et paroissiales pèsent leurs mots, jouent sur les sentiments, afin de bien sensibiliser le monarque à leur cause. Tout leur est bon pour obtenir des subsides du Prince, au point de clamer leur extrême pauvreté. Et pour bien retenir l'attention du Conseil de Roi, ils prennent soin d'envoyer cette lettre à trois reprises en une semaine. Pour quel résultat? Au cours de son périple politique dans le duché, à l'été 1518, François $1^{\mathrm{er}}$ refait le parcours de la duchesse Anne treize ans plus tôt, afin d'affirmer la continuité du pouvoir royal en Bretagne. On peut penser qu'il ait accédé aux demandes des paroissiens, comme l'avait d'ailleurs fait Anne en son temps car, même si le roi avait déjà donné vingt-cinq écus d'or l'année précédente, il est prouvé qu'il apporta son aide en 1529, distribuant cent vingt livres pendant dix ans. Or, 1529 pourrait bien ne constituer qu'un renouvellement du versement à la fin du délai habituel de dix ans, comme l'aurait été celui de 1518 après les dix années de versements promis par Anne en 1508.

Le nom de Rohan restait-il au moins un tant soit peu associé au couvent des Clarisses? Rien n'est moins sûr. Un acte royal du 4 octobre 1514 semble attester l'échec définitif du projet du vicomte de faire oublier l'origine ducale de cet établissement ${ }^{55}$. Les sœurs sont alors l'objet de menaces dont il est difficile de cerner les motifs. Le roi Louis XII rappelle à cette occasion, au nom de la duchesse Claude, que « les prédécesseurs de notre dite fille ducs et princes de ce dit pays sont fondateurs du couvent ". Et pour mettre un terme à toute velléité d'agressivité du voisinage, le roi demande de " mettre ou faire mettre et asseoir, si requis en estez, pannonceaulx et escuzons de nos armes aux huys, portes, maisons et habitations dudit couvent ". Ainsi paré des symboles royaux (ou peut-être des blasons mi-parti d'hermines, mi-parti de lys), qui oserait s'en prendre au couvent des dames de Sainte-Claire et qui se permettrait d'encore contester l'origine régalienne d'un tel lieu de dévotion?

\section{La figure de Rohan, de l'histoire à l'amnésie}

L'ensemble des éléments de cette enquête nous conduit logiquement à conclure au rôle central du vicomte Jean II de Rohan dans l'histoire de Dinan à la fin du Moyen Âge. Il a été le chef de la ville pendant presque trente ans (1488-1516), représentant des rois de France et de la duchesse

54. AudRAn-DELliez, Christiane, Un périple politique : François $I^{\text {er }}$ visite son duché de Bretagne en 1518, Mémoire de maîtrise, dactyl., Univ. de Paris I, 1990. Le roi a dû passer dans la cité entre le 30 septembre (il est alors au château de la Hunaudaye) et le 6 octobre 1518 où il arrive à Dol, sans doute avant de se rendre à Saint-Malo, suivant ainsi le parcours de la duchesse Anne treize ans auparavant. Catalogue des Actes de François $1^{\text {er }}$, 1887-1902, t. VIII, p. 426.

55. Bibl. mun. de Dinan, Archives des Clarisses, pièce E 8 bis. 
de Bretagne. Capitaine, il a délégué cette fonction militaire à un fidèle lieutenant, lequel commandait plusieurs dizaines de soldats présents dans la place, surtout au plus fort du conflit entre France et Bretagne. Lors de ses rares périodes de présence, il pouvait présider l'assemblée municipale, en lieu et place du procureur, maire avant l'heure choisi par les Dinannais. En tant que maître du domaine de Dinan, il faisait percevoir ses revenus par un receveur de confiance, dans et hors la ville, et bénéficiait de toutes les prérogatives inhérentes à son titre de seigneur de Dinan, notamment l'administration judiciaire. Membre éminent de l'une des dynasties les plus riches et influentes de la noblesse de Bretagne, il connut pourtant un échec dans notre ville, celui de n'avoir pu devenir le fondateur d'un couvent, dans une cité déjà fortement marquée par une forte présence des institutions mendiantes. Ce qui ne l'empêcha point de dépenser une part de "sa " richesse dans la pierre sacrée, d'abord par des donations au couvent des Clarisses, mais surtout par la fondation de la nouvelle église Saint-Malo de dinannaise. Mais alors, pourquoi cette présence n'a-t-elle jamais été vraiment reconnue dans la cité dinannaise?

D'abord, à la mort de Jean II de Rohan, sa famille perd le pouvoir sur la ville. Bien que François $1^{\text {er }}$ favorise l'exécution du testament du vicomte (au nom de " l'amour que portons à feu notre dit oncle et pour la perpétuité de son lignaige dont il attenoit à nous et à notre tres chere et tres amée compagne la reine ${ }^{56}$ "), en juin 1518 , le souverain fait don de la place de Dinan et des revenus de son domaine à Pierre de Laval, seigneur de Montafilant, pour le récompenser de sa bravoure lors des guerres d'Italie. Après trente ans entre les mains des Rohan, une autre famille bretonne prestigieuse prenait donc les rênes du pouvoir à Dinan pour vingt années ${ }^{57}$.

Le nom de Rohan allait bientôt incarner la présence protestante dans la province de Bretagne, puisque ses propres descendants deviendront d'actifs prosélytes de la nouvelle religion, à commencer par son petit-fils Henri $1^{\text {er }} 58$. La famille de Rohan n'est donc plus une référence avantageuse et fréquentable pour le sanctuaire paroissial et l'ensemble des habitants, surtout dans une ville tenue à la fin du Xvi ${ }^{\mathrm{e}}$ siècle par le duc de Mercoeur et ses catholiques zélés, puis résolument attachée au catholicisme par la suite ${ }^{59}$. Voilà qui a dû contribuer à rejeter dans l'oubli l'œuvre de Jean de

56. Le Page, Dominique, art. cit., p. 141. 51, Arch. dép. de Loire-Atlantique, B 51 $\mathrm{f}^{\circ} 199-200$

57. Le PAGE, Dominique, op. cit., p. 526-527. Guillaume Le Bascle fut maintenu à son poste malgré ce changement de capitaine et le conserva jusqu'en 1523. Il fut chargé du financement de réparations des fortifications de Dinan en 1520 et 1522.

58. CoRnetTe, Joël, Histoire de la Bretagne et des Bretons, Paris, Seuil, 2005, t. I, p. 457-459 pour le rôle important, voire central, des Rohan dans la naissance du protestantisme en Bretagne.

59. Sur Dinan à l'époque de Mercœur, voir le long développement de MonNIER, Mathurin, op. cit., p. 477-490. Une des preuves de la force du catholicisme à Dinan est le succès de la réforme tridentine catholique, en particulier la multiplication des couvents féminins au $\mathrm{XVII}^{\mathrm{e}}$ siècle. Se reporter à la brillante étude de OLLIVIER, Gilles, Les Couvents et la ville. Les fondations dinannaises du XVIr siècle à la révolution, Bannalec, 1985. 
Rohan. Pourtant, en 1636, le voyageur français Dubuisson-Aubenay décrit l'église de la paroisse Saint-Malo comme possédant un «beau et grand vaisseau de belle pierre tout achevé jusques aux voustes et combles qui est de bois et dont ceux de Rohan sont fondateurs y ayant leurs armes partout es piliers et vitres ${ }^{60}$ ». Bien qu'ayant beaucoup moins contribué au financement de l'édifice que les rois et reines de France, c'est encore à Jean de Rohan et à son lignage que reviennent la gloire et les honneurs de la fondation du sanctuaire, grâce à une présence quasi publicitaire de ses insignes nobiliaires. Cela prouve, d'une part, que tous les emblèmes de Jean II avaient survécu aux tensions iconoclastes qui ont affecté la ville lors des guerres de religion, dans la seconde moitié du Xvi ${ }^{\mathrm{e}}$ siècle ${ }^{61}$ et, d'autre part, que l'on savait toujours identifier le fondateur du sanctuaire plus d'un siècle après sa mort.

Mais ce sont sans doute les destructions de la période révolutionnaire, à la fin du XVIII ${ }^{\mathrm{e}}$ siècle, qui ont dû sonner le glas de la notoriété de Rohan à Dinan ${ }^{62}$. Situées aux voûtes de l'édifice et sur les piliers du chœur, ses armoiries hautement colorées étaient alors uniquement perçues comme des symboles de pouvoirs désormais arbitraires et révolus. Sur ordre de l'assemblée municipale, elles furent détruites en toute légalité. Sans la mutilation des armoiries de Jean II de Rohan, le personnage aurait sans doute plus attiré l'attention des historiens et généalogistes depuis le XIX ${ }^{\mathrm{e}}$ siècle et aurait ainsi trouvé sa vraie place dans la tradition historique dinannaise.

Ironie de l'histoire, c'est la figure de sa rivale Anne de Bretagne qui s'est imposée dans la mémoire collective de la ville, jusqu'à être exploitée à satiété à des fins touristiques ${ }^{63}$. Cette revanche posthume est sans doute à mettre sur le compte de quelques auteurs romantiques du XIX ${ }^{\mathrm{e}}$ siècle, tel Arcisse de Caumont qui, le premier en 1840, baptisa le Donjon de " palais de la duchesse Anne ${ }^{64}$ ». Cependant, la source de cette légende est probablement plus ancienne : elle prend corps chez certains chroniqueurs des XVI et XVII ${ }^{\mathrm{e}}$ siècles, à l'origine du mythe de la " bonne duchesse, " proche de son peuple breton et le défendant des convoitises des rois de France ${ }^{65}$.

60. DUBUISSON-AUBENAY, op. cit., p. 222.

61. Sur la présence protestante à Dinan, je renvoie à mon mémoire de maîtrise, op. cit., MARTIN, Hervé (dir.), Rennes II, 1989, p. 227-231.

62. Comble de malchance : en 1735 , les vitraux de l'église, y compris celui de la mâ̂tresse vitre armoriée par Rohan, n'ont pas résister à une violente tempête. MoNNIER, Mathurin, op. cit., p. 141 et 150.

63. Sur le site Internet de promotion touristique $w w w$.dinan.net, on peut même lire que l'on doit aux bienfaits de la duchesse la construction du beffroi municipal et de l'église Saint-Malo!

64. MonNIER, Mathurin, op. cit., p. 275-276. L'expression la plus couramment utilisée est celle de " château de la duchesse Anne ".

65. Mathurin MONNIER cite un chroniqueur, malheureusement sans mentionner son nom, qui affirmait qu'Anne " aimoit parcourir son duché de Bretagne, surtout pour aller se pencher sur le donjon de Dinan ». Sur l'histoire des représentations d'Anne de Bretagne, voir LE Fur, Didier, Anne de Bretagne : miroir d'une reine, historiographie d'un mythe, Paris, Guénégaud, 2000. 


\section{La revanche symbolique de Rohan}

En dépit du mécénat actif de Jean II de Rohan au profit de l'une des deux églises paroissiales, sa figure semble donc s'être irrémédiablement estompée de l'histoire de la cité. La notoriété de la reine Anne y est sans doute pour beaucoup, au même titre que la destruction à la Révolution de ses signes armoriaux jumelés à ceux de son épouse Marie de Bretagne. Pourtant, presque cinq siècles après sa disparition, l'ultime message laissé par le vicomte dans la ville a pu être mis à jour et identifié. En effet, à la jonction du déambulatoire et de la chapelle centrale du chœur gothique de ce même édifice, à l'entrée de sa chapelle privative, Jean II de Rohan a fait graver dans la pierre un véritable testament iconographique, sous la forme d'une discrète clef de voûte (Figure 6$)^{66}$.

Figure 6 - «Dieu, le diable et les sept péchés capitaux », clef de voûte de l'église Saint-Malo de Dinan, vers 1500

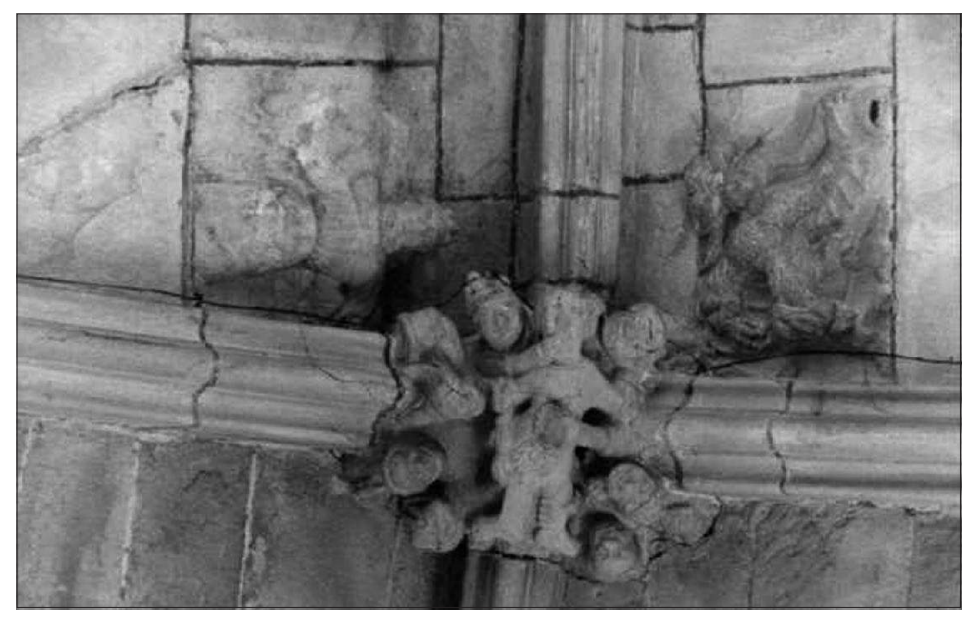

Au centre de ce haut-relief, un homme est allongé sur le dos, le bras droit contre le flanc, l'autre à demi tendu. Il est prisonnier de sept individus au faciès inquiétant, lesquels l'agrippent de toute part et s'acharnent sur une partie de son corps. Il s'agit de toute évidence d'une personnification des sept péchés capitaux permettant simultanément la représentation de la punition éternelle d'un pécheur en enfer, motif également visible à Batz et à Saint-Léry ${ }^{67}$. Mais cette œuvre s'en différencie par la présence symé-

66. Pour une analyse globale de cette œuvre inconnue jusqu'alors, je me permets de renvoyer à mon article " Jean II de Rohan et le diable à Dinan ", Le Pays de Dinan, p. 289317, t. XXVI, 2006.

67. Ibidem, p. 296 et 297. Ces deux œuvres sont de peu antérieures : probablement des décennies 1460-1470 pour Batz et de la fin des années 1470 ou du début de la décennie 1480 pour Saint-Léry. 
trique de deux personnages antagonistes : à la droite de la victime, Dieu le Père est figuré de face, dans une position hiératique, et illuminé par une blancheur écarlate; à sa gauche, le diable apparaît dans une teinte sombre et se contorsionne allègrement. Si le motif central du septénaire des vices existe donc en trois exemplaires dans toute la Bretagne, le triptyque « Dieu - diable - sept péchés capitaux " apparaît bien comme unique dans notre région, et sans doute au-delà.

Cette sculpture exceptionnelle doit être considérée avant tout comme une œuvre théologique complexe : elle reflète les convictions religieuses personnelles de son mécène, unanimement partagées par ses contemporains à l'époque, notamment ses craintes de l'enfer et de ses tourments, mais aussi ses espoirs d'une vie spirituelle en l'au-delà. C'est donc en toute logique que Jean II a fait apposer, à la droite de Dieu, son emblème personnel, le "A " surmonté d'une couronne fleuronnée ${ }^{68}$, que le Père semble protéger des violences infernales. Il faut remarquer que le vicomte n'a pas choisi de figurer ses armes familiales, " de gueules aux macles d'or ", mais son chiffre, ce qui apparaît logique dans une ouvre mettant en exergue la dimension individuelle du jugement.

Pourtant, ce symbole est depuis longtemps source d'interrogation pour les historiens. L'hypothèse la plus probable fait de ce "A couronné " l'initiale de la devise de Jean II " À Plus ». Mais il est tout aussi vraisemblable d'y voir une référence à son père le vicomte Alain IX de Rohan. En effet, Michel Nassiet a montré que le projet politique des Rohan, celui d'accéder au trône du duché de Bretagne, n'est pas uniquement le fait d'un individu, mais celui d'une lignée, se présentant comme apparentée en ligne masculine à la maison ducale ${ }^{69}$. En somme, le " $\mathrm{A}$ " serait un symbole de révérence filiale et de continuité dynastique. Pourtant, dans la structure de ce chiffre, deux détails ne sont pas sans étonner. D'une part, il ressemble trait pour trait au monogramme de la duchesse Anne, tel qu'il apparait par exemple dans les livres d'heures de la duchesse ${ }^{70}$. Cette similitude troublante a été interprétée

68. Bien qu'ayant subi les foudres destructrices des iconoclastes révolutionnaires en 1794, ce symbole peut encore être identifié sans ambiguïté. On le retrouve sur plusieurs bâtiments de la même époque notamment sur la façade gothique du château de Josselin, dont la construction est strictement contemporaine de la voûte de l'église Saint-Malo. L'auteur signale en outre que dans la chapelle Notre-Dame-de-Bonne-Encontre construite à Rohan en 1510, " les clefs de voûte sont ornées des armoiries maclées et du fameux A couronné ". Les consoles de pierre des piles est de la croisée sont agrémentées par des armoiries : l'une, " de gueules à 9 macles d'or ", blason des Rohan, est confortée par une deuxième " mi-partie de Rohan, six macles et mi-parti de Bretagne, deux hermines (rappelant l'union de Jean II avec Marie de Bretagne) ", Mussat, André, art. cit., 1997, p. 240-241; GiCQuel, Yvonig, op. cit., p. 450-453.

69. NASSIET, Michel, art. cit., p. 115-125. Il est aussi possible que le vicomte ait voulu y associer sa première fille née en 1485 et prénommée Anne, peut-être en l'honneur de la future duchesse à qui Jean II espérait faire épouser son fils aîné. Car ce prénom reste exceptionnel dans la lignée des Rohan.

70. Les "Petites Heures d'Anne de Bretagne ", vers 1500, BNF, Nouv. Acq. Latines 3027; Les « Grandes Heures d'Anne de Bretagne " de Jean BouRdichoN, vers 1508, BNF, ms lat. 
soit comme un hommage à la reine-duchesse, soit comme une ambiguïté amusante avec les noms de son père et d'Anne ${ }^{71}$. D'autre part, le choix d'une couronne à fleurons au sommet du A est plutôt troublant. En effet, sur un jeton en argent émis par la chambre des comptes de Jean II, c'est une couronne vicomtale qui est représentée au haut de l'écu, sous la forme de deux rangées de huit perles superposées. En revanche, celle qu'on distingue au sommet du " $\mathrm{A}$ " ne correspond manifestement pas au titre de vicomte mais représente clairement la couronne portée par les ducs de Bretagne depuis Jean $\mathrm{V}$ au début du $\mathrm{XV}^{\mathrm{e}}$ siècle $^{72}$.

Cette représentation du chiffre de Jean II de Rohan, association probablement volontaire entre l'initiale de la devise des Rohan, le prénom du père de Jean II et celui d'Anne de Bretagne, surmonté d'une couronne ducale, me semble participer de cette stratégie politique précédemment décrite par Michel Nassiet. Le " A " couronné se présente ainsi comme un signe polysémique : signe de remémoration de la génération précédente (celle du père), signe d'intégration de cette lignée à la maison ducale (commencée par le mariage de Jean II avec Marie de Bretagne) et si possible signe de substitution de la dynastie des Rohan à celle des Montfort (par le projet de mariage avec la jeune duchesse Anne). Pour autant, celui-ci me semble seulement revêtir une fonction mémorielle : si le " A " couronné apparaît sur tous les types de monuments des possessions de Rohan (militaires, civils, religieux), son apparition dans le mécénat de Jean II ne date que des années 1490, alors que l'espoir d'un tel mariage est devenu quasi irréalisable. Au final, la présence cumulée de ce " $\mathrm{A}$ " couronné à la clef de voûte axiale et des armes mi-parti Rohan-Bretagne sur les piliers et dans les vitraux de l'église Saint-Malo semble pour le moins relever d'une stratégie visuelle d'identification de son lignage à celui de Bretagne ${ }^{73}$.

Si l'on revient à présent à l'ensemble de cette sculpture religieuse, elle peut donner lieu à une interprétation politico-religieuse encore plus complexe et subtile. Cette commande de Rohan n'est pas une innovation. Deux autres exemplaires de cette image du pécheur torturé par les sept péchés capitaux sont donc recensés, également situées en Haute-Bretagne et datant du dernier tiers du Xve siècle : une autre clef de voûte, localisée dans le bascôté nord de l'église paroissiale Saint-Guénolé de Batz en Loire-Atlantique, remontant aux années 1460-1470 et un bas-relief en granit, visible à l'entrée du porche méridional de l'église paroissiale de Saint-Léry dans le Morbihan, réalisé peu avant 1486. Or, ces deux œuvres sont le fruit du mécénat ducal,

9474. Reproduction dans La Bretagne au temps des ducs, Catalogue de l'exposition de Daoulas, 1991, p. 169.

71. La première hypothèse est d'Yvonig GICQUEL, op. cit., p. 453, la seconde a été émise par André MussaT, art. cit., p. 240.

72. GiCQuel, Yvonig, op. cit., p. 216 et photographie hors texte; JonEs, Michaël, " En son habit royal : le duc de Bretagne et son image vers la fin du Moyen Âge ", dans Représentation, pouvoir et royauté à la fin du Moyen Âge, p. 253-275, Paris, Picard, 1995.

73. Ce choix ne s'explique pas par des raisons financières, puisque aucun don de la part de son épouse Marie n'est signalé dans les archives. 
en la personne de François II à Saint-Léry (associé à l'évêque de Saint-Malo Jean L'Épervier) et peut-être sa première épouse Marguerite de Bretagne à Batz. Jean II de Rohan s'est donc probablement inspiré d'un motif religieux préexistant, mais en y ajoutant une signification politique, afin d'y faire figurer les prétentions de sa lignée à la couronne ducale.

Car sur les sculptures de Batz et de Saint-Léry, le pécheur est représenté portant une couronne, tenue par un monstre figurant le péché d'orgueil ${ }^{74}$. Sur la sculpture de Dinan, le pécheur en est dépourvu. La seule couronne visible est celle située au-dessus du chiffre de Rohan, le "A " stylisé. Tout se passe comme si le vicomte avait voulu opérer un transfert de ce symbole ducal à son profit. Il s'agirait, par un procédé visuel, de la réalisation virtuelle de son projet politique de captation de la couronne ducale au profit de sa lignée. En poussant le raisonnement à l'extrême, ne peut-on raisonnablement aller jusqu'à penser que Jean II de Rohan ait eu la tentation de faire représenter son plus intime ennemi politique endurant les tourments de l'enfer, à savoir le défunt duc de Bretagne François II? Celui-ci n'a-t-il pas réussi à contrarier les projets d'accession au trône de Bretagne du lignage des Rohan?

Plus étonnant encore, ce conflit symbolique a peut-être été déclenché par le duc en personne. Rappelons que Jean II de Rohan revendiquait haut et fort le titre de "premier baron de Bretagne " dans les années 1480, alors même qu'il croupissait dans les geôles ducales... Réalisée dans ces mêmes années, la sculpture de Saint-Léry, une paroisse située dans le domaine ducal mais à la lisière des fiefs de Rohan, exhibe un pécheur nu, ne portant qu'une couronne... de vicomte ${ }^{75}$ ! Le haut-relief de Dinan ne serait-il que le pendant du bas-relief de Saint-Léry? Jean II de Rohan n'aurait-il fait que répondre à une provocation symbolique et spirituelle du duc François II? Le conflit politique entre les deux Grands de Bretagne aurait-il pris une dimension théologique? À la condamnation à l'enfer du vicomte par François II,

74. On retrouve aussi cette symbolique de la couronne à Batz. Le monstre placé audessus de la tête du pécheur de Batz présente nettement une tête de lion ou peut-être sa représentation négative, celle du léopard. Il ne manifeste pas de gestes agressifs à l'égard de l'homme : comme à Saint-Léry, il se contente de poser ou maintenir un objet sur son chef, en l'occurrence une couronne. La symbolique du lion, devenu le " roi des animaux " depuis le XIII ${ }^{\mathrm{e}}$ siècle, la présence d'un objet essentiel du pouvoir royal et la position du démon à proximité de la tête, autant d'indices d'une personnification du péché d'orgueil, d'ailleurs confirmé par son inscription en français sur un phylactère placé à cet endroit. Ce péché ne se manifeste-t-il pas par " une volonté indue d'élévation et de domination sur les autres? C'est pourquoi il est essentiellement le péché des puissants ". Citation de Jérôme BASCHET, "Les sept péchés capitaux et leurs châtiments dans l'iconographie médiévale ", dans CASAGRANDE, Carla VECCHIO, Silvia, Histoire des péchés capitaux au Moyen Âge, Paris, Aubier, 2003, p. 378. Sur l'histoire symbolique du lion, je renvoie à Michel PASTOUREAU, "Le sacre du lion ", Une histoire symbolique du Moyen Âge occidental, Paris, Le Seuil, 2004, p. 49-64.

75. L'exemplaire représenté sur le bas-relief de Saint-Léry s'apparente en effet au modèle de la couronne à perles décrit par Paola RAPELLI, Symboles du pouvoir, Paris, Hazan, 2005, p. 27 : il s'agit soit de la couronne d'un comte comportant seize perles, soit de la couronne d'un baron formée d'une spirale de perles. 
Jean II aurait-il répondu par la même peine infligée au duc, tout en signalant la protection divine dont lui jouissait?

Quoi qu'il en soit, pendant presque trente ans, la cité de Dinan est restée sous le contrôle du vicomte de Rohan, avec l'aval des souverains successifs. Ce cadeau royal lui a permis de poursuivre sa politique de mise en valeur de son lignage, en particulier par un mécénat qui entendait faire de l'ombre à celui du duc François II. Recueillant les larges revenus de la cité et de son plat pays, s'appuyant sur des hommes de confiance, un temps encore il a tenté de concrétiser son grand projet politique : faire accéder son lignage au trône de Bretagne. Ayant compris son échec suite au mariage de la duchesse Anne avec le roi de France, il se résigne à ne faire de Dinan qu'une vitrine de sa puissance. Lors de ses passages dans la cité, notamment lorsqu'il y accompagne à deux reprises au moins la duchesse-reine, il peut faire admirer le résultat de son mécénat, notamment le chevet plutôt original du sanctuaire paroissial de Saint-Malo, ou encore parrainer les cloches du nouveau beffroi. Juste retour des choses pour les bourgeois de Dinan car eux aussi ont bénéficié de cette mainmise du vicomte de Rohan. Pour l'élite locale, c'est une opportunité unique d'accéder enfin à des charges municipales et des offices rémunérateurs, en entrant dans le réseau clientéliste du vicomte; pour les paroissiens et le clergé de Dinan, c'est l'occasion de bénéficier à moindre coût d'un mécénat religieux novateur et de faire monter les enchères du financement du nouvel édifice paroissial, en s'adressant alternativement au vicomte ou à la duchesse-reine et ses époux. C'est enfin la garantie pour tous d'une protection renforcée grâce à une garnison permanente aux ordres de Jean II, particulièrement lors des années de troubles. Néanmoins, progressivement, la figure de Rohan s'est estompée de l'histoire de Dinan et de la mémoire des Dinannais pour laisser triompher celle de sa rivale, la duchesse Anne. Pourtant, dans cette ville, le vicomte a laissé l'une des œuvres les plus originales de son mécénat, une commande subtile, qui lui a permis d'affirmer et de concilier obsession politique et inquiétude eschatologique : c'est en effet à Saint-Malo de Dinan que Jean II de Rohan a pu symboliquement réaliser les deux objectifs de son existence, associer son lignage au trône ducal, sauver son âme de pêcheur. 


\section{RÉSUMÉ}

L'objet de ce travail est de mettre en relation l'un des grands personnages de la Bretagne à la fin du Moyen Âge et une importante ville du duché sur les plans démographique, économique et stratégique. Le vicomte Jean II de Rohan reçoit la capitulation de Dinan lors du conflit franco-breton en 1488 et en garde le contrôle jusqu'à sa mort en 1516, avec l'aval des souverains successifs. Cette cité lui offre une opportunité supplémentaire de poursuivre son action politique d'accession de son lignage au trône de Bretagne : pour ce faire, il instaure un mécénat qui tend à se substituer à celui du duc François II. S’il le supplante aisément dans la fondation de l'église paroissiale Saint-Malo de Dinan, il essuie par contre un échec dans sa tentative auprès du couvent des Clarisses. Il est vrai qu'il se heurte en permanence aux interventions répétées de l'héritière du duc, Anne de Bretagne, et de ses époux royaux dans la cité. Pour financer ses dépenses monumentales, Rohan dispose des revenus réguliers du domaine de Dinan, dont il s'assure un contrôle exclusif en plaçant de fidèles agents aux postes clés. Ce véritable réseau clientéliste, composé d'officiers ducaux et de notables locaux, ne se prive pas d'asseoir son pouvoir sur la cité et d'accroître sa fortune. Quant à la population dinannaise, elle a aussi bénéficié de cette mainmise du vicomte de Rohan, à travers un mécénat novateur et une protection renforcée. Néanmoins, progressivement, la figure de Rohan s'estompe de l'histoire de Dinan et de la mémoire des Dinannais pour laisser triompher celle de sa rivale, la duchesse Anne. Mais c'était sans compter sur la volonté du vicomte de laisser une empreinte durable dans la cité, dépassant la stricte échelle spatio-temporelle de Dinan à la fin du Moyen Âge. Grâce à une commande artistique originale, il a été en mesure de concilier son obsession politique et ses inquiétudes eschatologiques : à Saint-Malo de Dinan, il a pu symboliquement associer son lignage au trône ducal et sauver son âme.

\section{ABSTRACT}

The subject of this article is linking one of the greatest men of Brittany in the late Middle Ages to an important city of the dukedom as far as demography, economy and geography were concerned. In 1488, with the backing of the successive kings, Viscount Jean II of Rohan received the capitulation of the city of Dinan and kept control over it till he died in 1516. The city first enabled him to follow a policy according to which his sole lineage could ascend the throne of Brittany; so that he could achieve this, he established a patronage to compete with that of François II. If he easily supplanted him with the building of Saint-Malo de Dinan parish church, the completion of the building of the Franciscan Sister's Convent conversely was a blow. As a result he constantly faced Ann of Brittany's permanent interventions and those of her royal husbands in the city. To finance the huge expenditures, Rohan got regular loans from the property of Dinan and over which he had an exclusive control by putting faithful agents into key positions. This real clientist network of people composed of ducal officers and local notables took advantage of the situation to settle their power over the city as well as increase their wealth. As far as the inhabitants in Dinan were concerned, they benefited a little from Viscount of Rohan's stranglehold thanks to an innovative patronage and strengthened protection. Rohan's fame, however, gradually faded from people's memories in Dinan, as well as from the history of the city, to favour that of Duchess Ann. But it was without relying on the Viscount's will to leave an everlasting mark in the city, going beyond the strict spatial and temporal scale of Dinan in the late Middle Ages. Thanks to an uncommon artistic commission, he managed to reconcile his political obsession with his eschatological worries: in SaintMalo de Dinan, he succeeded in creating a symbolical link between his sole lineage on the ducal throne and saving of his soul. 